\title{
The liquefied natural gas infrastructure and tanker fleet sizing problem
}

\author{
Koza, David Franz; Røpke, Stefan; Molas, Anna Boleda
}

Published in:

Transportation Research. Part E: Logistics and Transportation Review

Link to article, DOI:

10.1016/j.tre.2017.01.003

Publication date:

2017

Document Version

Peer reviewed version

Link back to DTU Orbit

Citation (APA):

Koza, D. F., Røpke, S., \& Molas, A. B. (2017). The liquefied natural gas infrastructure and tanker fleet sizing problem. Transportation Research. Part E: Logistics and Transportation Review, 99, 96-114. https://doi.org/10.1016/j.tre.2017.01.003

\section{General rights}

Copyright and moral rights for the publications made accessible in the public portal are retained by the authors and/or other copyright owners and it is a condition of accessing publications that users recognise and abide by the legal requirements associated with these rights.

- Users may download and print one copy of any publication from the public portal for the purpose of private study or research.

- You may not further distribute the material or use it for any profit-making activity or commercial gain

- You may freely distribute the URL identifying the publication in the public portal 


\title{
An exact solution approach for the liquefied natural gas infras- tructure sizing and tanker routing problem
}

\author{
David Franz Koza ${ }^{\mathrm{a} *}$, Stefan Ropke ${ }^{\mathrm{a}}$, Anna Boleda Molas ${ }^{\mathrm{b}}$ \\ ${ }^{a}$ DTU Management Engineering, Technical University of Denmark, Produktionstorvet 424, 2800 Kgs. Lyngby, \\ Denmark \\ ${ }^{\mathrm{b}}$ L'Oréal Danmark A/S, Havneholmen 25, 1561 Copenhagen V, Denmark
}

\begin{abstract}
In this work we present a combined infrastructure sizing and tanker routing problem in the liquefied natural gas (LNG) business that is based on a business case study with a major liner shipping company. The decision problem is of strategic nature and consists of selecting the LNG storage capacity at each port of demand as well as defining the size and number of tankers and their shipping routes used to transport the LNG from its source port to the ports of demand. The goal is to minimize long term investment and operational costs.

The introduction of global limits on sulphur and nitro oxide emissions has increased the interest in LNG as an alternative fuel for vessels, including container ships. As the global LNG infrastructure is still underdeveloped, it requires both strategic investment as well as tactical routing decisions to make LNG available at the points of demand. We propose mathematical models for determining the capacities of the necessary LNG infrastructure as well as the size and routes of LNG tankers needed for transportation.

Two models are presented. The first, non-linear model represents an intuitive formulation of the optimization problem, but is hard to solve. The second formulation is based on the set-partitioning model and is very attractive from a computational point of view. First, a set of partial solutions is generated through enumeration. In the second step a set-partitioning problem is solved to determine the best combination of the previously generated partial solutions. Results for the case study are presented and an extensive sensitivity analysis is conducted to account for the limited predictability of key parameter values, to analyse the robustness of the obtained solutions and to derive basic decision rules.

Keywords: Liquefied Natural Gas (LNG) as fuel, liner shipping, infrastructure planning, tanker routing, mixed integer programming
\end{abstract}

\section{Introduction}

In 2008 the International Maritime Organization (IMO), a specialized agency of the United Nations, has introduced new regulations for the prevention of pollution from ships that aim at reducing the emission of sulphur oxides, amongst others (International Maritime Organization, 2008). The regulations apply in so-called Emission Control Areas (ECA) since 2015 already and will become binding globally in 2020 or, if compliance appears to be impossible in 2018, in 2025 . Currently the majority of ships (80-85\%, Chryssakis et al., 2014), including container vessels, are run on heavy fuel oil (HFO). As a consequence of the new limits on sulphur emissions, ships will no longer be able to operate as of today, because emissions due to the use of HFO exceed the limits.

In various industrial strategic papers and research studies (see e.g. Andersen et al., 2013; Rozmarynowska and Oldakowski, 2012; Chryssakis et al., 2014) three viable solutions to meet the new requirements have been identified. The first one are exhaust gas aftertreatment systems as e.g. scrubbers. The installation of scrubbers, however, is costly and requires additional space on the ship. Further, they can increase the fuel consumption of a vessel by 2-3\% (Chryssakis et al., 2014). The second and most straightforward solution is the use of cleaner marine diesel oil (MDO) or marine gas oil (MGO), as these can usually be used without the need of any modification to the vessels. MDO and MGO are, however, about 1.5-2.0 times more expensive than HFO, with prices expected to increase even further once the sulphur emission limits apply globally.

The third solution is the one that motivates our study and considers liquefied natural gas (LNG) as an alternative fuel. LNG is natural gas that is converted to liquid form by cooling it down to approximately $-162^{\circ} \mathrm{C}\left(-260^{\circ} \mathrm{F}\right)$. It is the cleanest form of fossil fuels and if used 


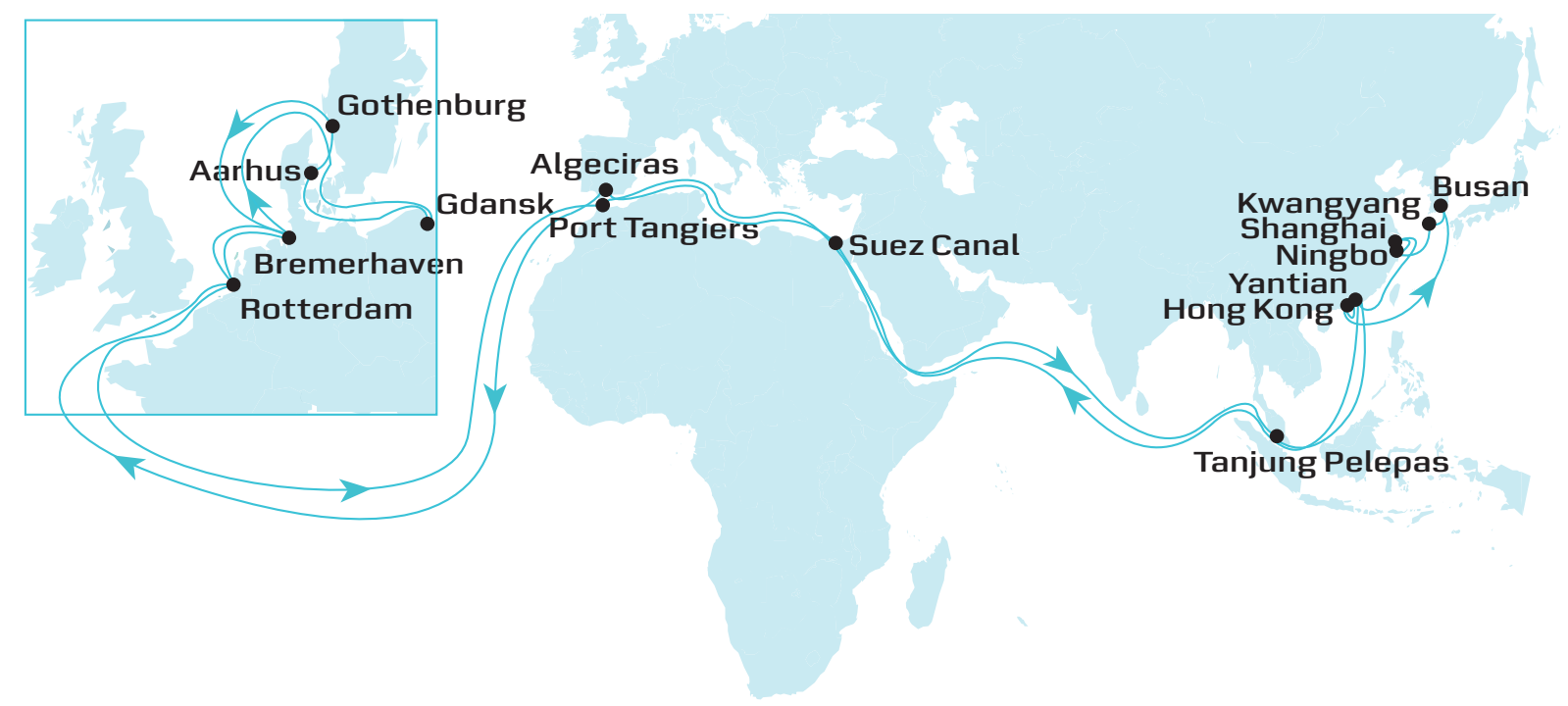

Figure 1: Example of a liner shipping service between Asia and Europe. The whole round trip takes 12 weeks and hence 12 vessels are operating on the service to ensure a weekly frequency.

to fuel ships, no further measures are needed to satisfy the new regulations concerning the emission of pollutants. LNG is considered a realistic option for deep sea trades in the long term, particularly for liner trades (Lloyd's Register, 2012).

Liner shipping networks consist of cyclic shipping routes, called services, that are operated periodically. Figure 1 shows an example liner shipping service that connects Asia and Europe. The individual services are connected through ports, where cargo can be transshipped between different services, and thus provide an extensive, wide-ranging transportation network. Typically each port on a service will be visited once per week and the container liner company publishes the weekly berthing time for each port. As a single round trip can take several weeks, each service is operated by a corresponding number of container vessels. The structure and way of operating container shipping networks is very similar to that of buses in public transport, with the containers being the equivalent of passengers.

The current lack of LNG infrastructure for marine bunkering and the uncertainty about future availability still is a major drawback of using LNG as a fuel for liner shipping companies. This work is motivated by and based on a case study with a major liner shipping company that considers filling that gap by building up and operating the needed infrastructure by themselves. The study forms the basis of a future scenario in which the company uses LNG fuelled container vessels on some of their services.

The liner shipping company is responsible for the transport of LNG to predetermined ports where container vessels will refuel. The transport is done via sea using special purpose LNG tankers. The tanker fleet needs to be ordered or chartered by the company. Furthermore, the infrastructure at the majority of the ports of demand will be built and run by the liner shipping company. The problem is of strategic nature with a time horizon of 2-15 years and combines strategic infrastructure and tanker investment decisions with tactical tanker routing and inventory management decisions. The lack of existing infrastructure allows to simultaneously optimize strategic investment decisions and interdependent tactical decisions.

Strategic infrastructure planning and tactical planning of operations have traditionally been looked at separately. For tactical and operational problems, the infrastructure and the fleet of vehicles is usually fixed to a large extent. Furthermore, strategic decisions often do not solely depend on quantifiable parameters but are subject to many qualitative arguments (legal issues, local regulations, political decisions, etc). This work aims at providing decision rules of thumb and identifying important relationships between operational/tactical and strategic decisions for 
the problem studied. The presented models also allow to evaluate manually developed solutions and their sensitivity to changes in input parameters. Hence, an important requirement of our industrial collaborator towards the solution method are fast running times that allow to evaluate large numbers of different scenarios within reasonable time.

The class of maritime inventory routing problems (MIRPs) is the one closest to the problem addressed in this work. In fact, if the strategic infrastructure and tanker investment decisions were fixed, the remaining problem would classify as a MIRP. The first studies on the MIRP are by Christiansen and Nygreen (1998) and Christiansen (1999) and deal with the production, shipping and inventory management of ammonia. Since then, most of the contributions are motivated by some specific application. Furman et al. (2011) present a mixed-integer programming formulation for vacuum gas oil routing and inventory management. Agra et al. (2013) and, more recently, Agra et al. (2015) consider a MIRP in the fuel oil distribution business, with the latter contribution assuming sailing and port times to be stochastic. An overview of maritime inventory routing problems together with examples of applications can be found in Christiansen and Fagerholt (2009). Several contributions to the MIRP literature with applications in the LNG business have been made during the recent years. Grønhaug and Christiansen (2009), Grønhaug et al. (2010) and Andersson et al. (2016) present different exact solution approaches for an LNG inventory routing problem. Rakke et al. (2011), Stålhane et al. (2012), Halvorsen-Weare and Fagerholt (2013), Halvorsen-Weare et al. (2013), Goel et al. (2012) and Rakke et al. (2015) and Andersson et al. (2015) have developed exact and heuristic solution approaches for LNG annual delivery program planning problems. The paper by Andersson et al. (2010) provides a general description of the LNG supply chain and presents two related problems. What these research studies have in common is that they address tactical or operational problems with planning horizons of at most several months. Different to our work, the onshore infrastructure and the available fleet of LNG tankers is generally assumed to be given and fixed. By contrast, the strategic infrastructure and fleet investment decisions play a key role in the problem. We believe that including these decisions change the nature of the problem significantly compared to existing MIRPs.

A study closer to our work is the one by Jokinen et al. (2015). They present a mixed integer linear programming model that aims at minimzing the cost of a small-scale LNG supply chain in southern Finland, including both annual terminal investment as well as transportation costs. LNG needs to be distributed from a large regasification terminal to several inland consumers, using both sea and land based transport through smaller satellite LNG terminals along the coast and LNG trucks that connect the ports with the points of demand. Even though the focus and scale of their study differ significantly from our work, their results underline the importance of simultaneously considering strategic investment and tactical routing decisions in the still underdeveloped LNG business.

The significant number of recently published industrial studies and surveys that address the feasibility and prospects of LNG as a fuel in deep sea container shipping demonstrate the practical relevance of the problem studied (e.g. DNV-GL, 2014; Andersen et al., 2013; Lloyd's Register, 2012, 2014).

The contribution of this paper is twofold: firstly, we introduce the infrastructure sizing and tanker routing problem that combines strategic and tactical decisions. We present two mathematical models for solving the problem, with the latter formulation allowing very short solution times. Secondly, we report computational results from a real-life case study. The results show that it pays off to optimize for strategic and tactical decisions simultaneously, because in the long perspective transportation costs can be reduced significantly through optimal investment decisions.

In Section 2 the problem is described in detail and corresponding assumptions are introduced. In Section 3 we present an arc-based model for conceptual reasons and derive a path based formulation that is used for the computational tests. The computational results are reported 


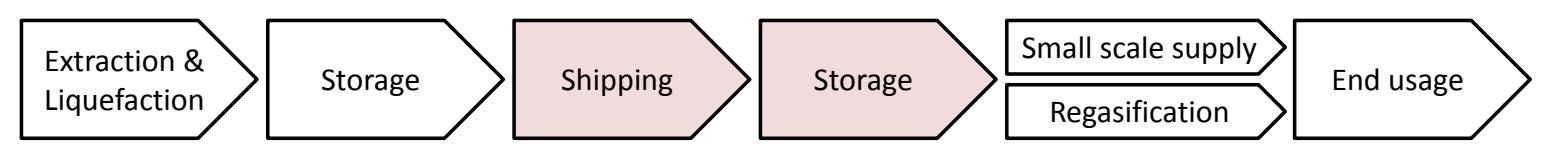

Figure 2: The LNG supply chain and the part considered in this work (following Andersson et al., 2010)

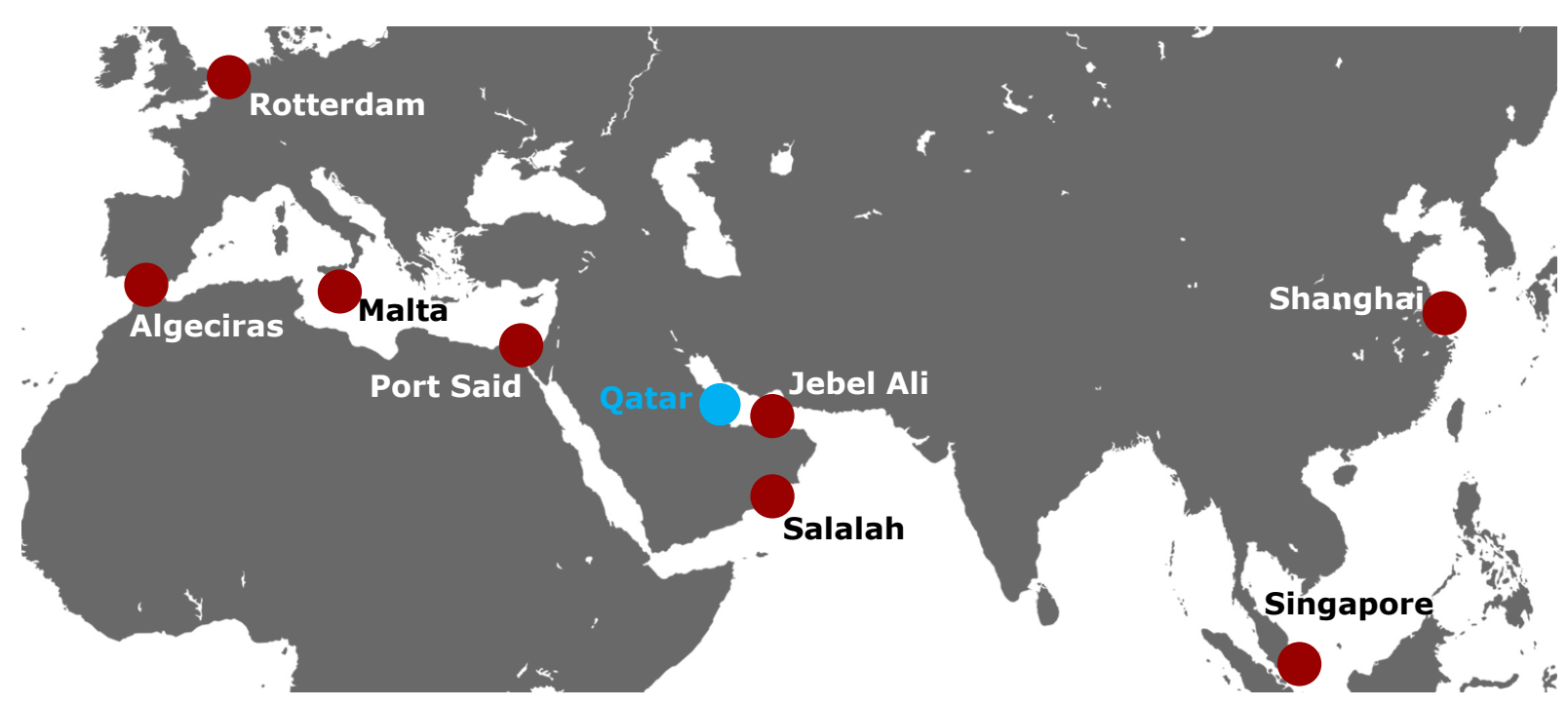

Figure 3: Planned refuelling stops.

in Section 4, together with a detailed description of the underlying data and cost. Concluding remarks are given in Section 5.

\section{Problem description}

The typical LNG supply chain in a maritime context can be described as follows (see e.g. Andersson et al., 2010): natural gas is extracted, purified and then turned into liquefied natural gas at a designated liquefaction plant. After liquefaction the LNG is stored in full-containment tanks that keep the gas in its liquefied state. It is then transported by special-purpose LNG tankers to its destination terminal, where it is unloaded into onshore storage tanks again. Nowadays most of the LNG is regasified at its destination and then distributed to end customers via pipelines or trucks. In our scenario, however, a large portion of the liquefied natural gas would finally be used to fuel container vessels (see Figure 2). The container ships would either be refuelled at ports with LNG bunkering facilities or by LNG bunker vessels.

All the LNG needed at the ports of demand in this study is considered to be extracted as natural gas (NG) and transformed into LNG at liquefaction plants by a third party in Qatar, the world's largest producer of LNG. In Qatar the LNG is loaded onto LNG tankers that are either owned or chartered by the liner shipping company. The destinations of the LNG are ports located along the route Asia-Europe. The number of ports that shall provide LNG refuelling services and their locations have been set by the company, based on their estimates of how large the LNG tanks on the container vessels will be and how often the ships will have to refuel. Generally, it is expected that LNG fuelled container vessels refuel more often because of the lower energy content per unit of volume. Depending on the LNG tank type used, the compartment housing of the tanks can be up to four times that of HFO tanks of equivalent energy content (Hannula et al., 2006). The eight ports at which onshore LNG storage tanks are planned to be built are shown in Figure 3. The rather large number of ports is explained by the fact that the ports will provide refuelling facilities to several liner shipping services along the Asia-Europe trade but also serve external small scale demand. The scenario of LNG produced onshore in Qatar 
and transported to Europe via LNG carriers has also been used in other studies (Chryssakis et al., 2014). The shipping of LNG from the port of supply to multiple ports of demand and the inventory management at the destination ports are part of the decision problem considered in this work.

The annual amount of LNG bought in Qatar equals the annual demand at all ports as estimated by the liner shipping company. The company's demand estimates cover the demand for LNG as fuel for its container vessels, but may also include some spot market demand at the ports. The company is responsible for carrying the LNG from Qatar to the ports of demand. In accordance with the cyclicity of container shipping services, a constant demand for LNG over time is assumed at the ports of demand. On an operational level fluctuations in demand may occur, for example, because of unplanned deviations from the route due to bad weather or delayed port operations that require the vessels to speed up. Given the assumption of a stable demand for LNG in the medium term, we will assume periodic shipping routes for the LNG tankers as well.

The maritime inventory routing problem that is part of the overall problem considered here can thus be classified as a single product MIRP with a single loading port, multiple unloading ports and constant production and consumption rates. Due to the strategic nature of the problem considered, we neglect the loss of LNG because of boil-off.

In addition to the tactical routing and inventory management decisions, the problem contains two strategic decision problems: first, the choice of the fleet of LNG tankers and, second, the determination of the onshore infrastructure capacity at the receiving ports.

We assume that LNG tankers of any capacity up to Q-Max size $\left(265000 \mathrm{~m}^{3}\right)$, the largest LNG tankers currently in operation, are available. The LNG tankers can either be owned or chartered by the company, in both cases we assume a charter rate based on the tanker capacity. It is worth noting that there exist tanker types that suffer from sloshing (see e.g. Gavory and De Seze, 2009). Sloshing is the motion of the liquid LNG inside the tanks and can create high pressure loads on the tank surface up to damaging it. As it mainly occurs in partially loaded membrane type tanks, they are generally filled to levels below $10 \%$ or above $70 \%$ of the tank height in order to reduce sloshing impacts (Kuo et al., 2009; Delorme et al., 2005). Research that aims at fully understanding and preventing the effects of sloshing in LNG tankers is still ongoing. We will address the issue by defining scenarios that remain feasible under the limitation of tankers being either fully loaded or empty.

The second strategic decision addresses the infrastructure and its capacity that has to be built up at the ports considered in this study (see Figure 3). At some of these ports LNG infrastructure already exists: the Maasvlakte LNG terminal at the port of Rotterdam, which opened in 2011, is considered a starting point of a small scale LNG supply chain in Northwest Europe (Drewry Maritime Research, 2012). Four storage tanks of $180000 \mathrm{~m}^{3}$ and two jetties capable of handling Q-max tankers exist. A break bulk terminal that allows small scale supply of LNG to bunker barges is planned to enter operations in the end of 2016. The Shanghai LNG terminal at Yangshan port has three $160000 \mathrm{~m}^{3}$ LNG tanks and is capable of handling tankers of up to $200000 \mathrm{~m}^{3}$ capacity. The Singapore LNG terminal has three $180000 \mathrm{~m}^{3}$ LNG tanks (working capacity) and jetties for tankers of up to $265000 \mathrm{~m}^{3}$ capacity available. We assume, however, that all the infrastructure capacity required by the liner shipping company will have to be newly constructed at each of the ports for two reasons: first, the capacities at the ports with existing infrastructure are expected not to be sufficient to additionally handle the demands estimated by the company. The demand for LNG in Rotterdam estimated by the company, for example, would already make up around $85 \%$ of the terminal's capacity for small scale distribution when put into operation at the end of 2016 (Gate terminal, 2014). The second reason is that many existing LNG terminals simply do not provide an LNG bunkering option. Adding an LNG bunkering option is not straightforward, because many additional regulations regarding design safety and separation distances have to be satisfied. Furthermore, most of the 
existing LNG terminals are designed for low frequency, high volume transfers (DNV-GL, 2014).

We are primarily interested in the interplay of operational cost and investment cost under different scenarios. Short-term solutions as e.g. floating LNG storages, i.e. anchored LNG tankers used as close-to-shore storages, have also been discussed with the company but neglected in this study as they are mainly considered as a bridging solution.

As a summary, the key decisions to take consist in choosing the right onshore infrastructure capacities at the receiving ports and the right tanker fleet to deliver the LNG, both in terms of capacities. Simultaneously, a routing problem is solved such that the routes both satisfy the annual demand and are compatible with the chosen infrastructure options. The goal is to minimize the combined annual investment cost and operational cost in the long and medium term.

\section{Modelling and solution method}

We first present a non-linear arc-based formulation of a simplification of the underlying problem (sec. 3.1). We do not solve the arc-based model, but use it to conceptually introduce the problem to the reader. A very similar model has been tested by Boleda Molas (2015) but turned out to be hard to be solved in reasonable time. In Section 3.2 we derive a path-based model that allows for a higher level of details incorporated and also has a much simpler structure. The path based formulation relies on the prior generation of partial solutions, which will be described in Section 3.3.

\subsection{Arc-based model formulation}

Let $\mathcal{P}$ denote the set of ports of demand and $\mathcal{P}^{0}=\mathcal{P} \cup\{0\}$ be the set of all ports, including the port of supply, indexed by 0 . Let $\mathcal{G}\left(\mathcal{P}^{0}, \mathcal{A}\right)$ denote the corresponding complete graph with the node set $\mathcal{P}^{0}$ and the set of directed $\operatorname{arcs} \mathcal{A}$, representing sailing legs between all pairs of ports. The set of available LNG tanker types is given by $\mathcal{M}$ and indexed by $m$. Each tanker type is uniquely defined by its capacity $q_{m}$.

The annual demand for LNG at each port of demand $i \in \mathcal{P}$ is denoted by parameter $d_{i}$. We assume a constant sailing speed for each tanker type and hence can derive a time parameter $t_{i j m}$ for each sailing leg $(i, j)$ and tanker type $m$. The time includes the sailing time between ports $i$ and $j$ but also pilot-out and pilot-in times at the origin and destination port and the time for loading LNG (if $i=0$ ) or unloading LNG (if $i \neq 0$ ) at the origin port $i$.

Different cost factors are considered in the model. The annual tanker type dependant charter cost is denoted by $c_{m}^{\text {ch }}$ for each tanker used, independent of its utilization. The fuel $\operatorname{cost} c_{i j m}^{\text {fuel }}$ is defined per sailing leg and tanker type and also includes cost for in-port fuel consumption at the origin port. For each port visit, a port and tanker type dependant port call cost $c_{i m}^{\text {port }}$ is added. Similarly, for each use of a sailing leg that includes a canal, a tanker type dependant canal fee $c_{i j m}^{\text {canal }}$ is added. In general the cost for onshore infrastructure at a location $i$ is non-linear, with the additional cost per extra unit of capacity decreasing for larger capacities, reflecting economies of scale. The used infrastructure cost function $\sum_{i \in \mathcal{P}} \bar{c}_{\text {ref }}\left(\frac{y_{i}}{\bar{y}_{\text {ref }}}\right)^{\delta}$ depends on the capacity $y_{i}$ and is based on a given reference project with cost $\bar{c}_{\text {ref }}$ and capacity $\bar{y}_{\text {ref }}$ and an estimated parameter $\delta$. The capacity itself needs to be sufficient to receive the quantity of LNG unloaded at each tanker visit plus some relative buffer capacity $B$. Note that the annual cost for the onshore infrastructure includes both capital as well as operational expenditures. A detailed description is provided in Section 4.2. In order to keep the arc-based model simple, we neglect the inventory cost for LNG on tankers and in the storage tanks at the ports of demand, but will include them in the path-based formulation.

Since we assume that the demand of each port is fulfilled by exactly one tanker route, the output of the model will be at most $|\mathcal{P}|$ routes. We let $\mathcal{R}=\{1, \ldots,|\mathcal{P}|\}$ be the index set of the 
routes resulting from the model. We can now define the binary decision variables $x_{i j r m}$ that denote whether tanker type $m$ is used on sailing leg $(i, j) \in \mathcal{A}$ in route $r$ or not. The frequency of each route, i.e. how often the route is operated per year, is represented by the continuous decision variable $f_{r}$. The continuous variable $y_{i}$ denotes the infrastructure capacity at port $i$, on which the infrastructure cost at a port depends on. The number of tankers of type $m$ needed on route $r$ is given by the integer decision variable $n_{r m}$.

The problem can then be formulated as follows:

$$
\begin{aligned}
& \min \sum_{r \in \mathcal{R}} \sum_{m \in \mathcal{M}} c_{m}^{\mathrm{ch}} n_{r m}+\sum_{i \in \mathcal{P}^{0}} \sum_{j \in \mathcal{P}^{0}} \sum_{r \in \mathcal{R}} \sum_{m \in \mathcal{M}} c_{i j m}^{\mathrm{fuel}} x_{i j r m} \\
& +\sum_{i \in \mathcal{P}} \sum_{j \in \mathcal{P}^{0}} \sum_{r \in \mathcal{R}} \sum_{m \in \mathcal{M}} x_{i j r m} f_{r}\left(c_{i m}^{\text {port }}+c_{i j m}^{\text {canal }}\right)+\sum_{i \in \mathcal{P}} \bar{c}_{\text {ref }}^{\text {inf }}\left(\frac{y_{i}}{\bar{y}_{\text {ref }}}\right)^{\delta} \\
& \text { s.t. } \quad \sum_{j \in \mathcal{P}} \sum_{r \in \mathcal{R}} \sum_{m \in \mathcal{M}} x_{i j r m}=1 \quad \forall i \in \mathcal{P} \\
& \sum_{j \in \mathcal{P}^{0}} x_{j i r m}-\sum_{j \in \mathcal{P}^{0}} x_{i j r m}=0 \quad \forall i \in \mathcal{P}, r \in \mathcal{R}, m \in \mathcal{M} \\
& \sum_{i \in S} \sum_{j \notin S} \sum_{r \in \mathcal{R}} \sum_{m \in \mathcal{M}} x_{i j r m} \geq 1 \quad \forall S \subseteq \mathcal{P},|S| \geq 2 \\
& \sum_{j \in \mathcal{P}} \sum_{m \in \mathcal{M}} x_{0 j r m} \leq 1 \quad \forall r \in \mathcal{R} \\
& \sum_{i \in \mathcal{P}} \sum_{j \in \mathcal{P}^{0}} \sum_{m \in \mathcal{M}} \frac{x_{i j r m} d_{i}}{q_{m}}=f_{r} \quad \forall r \in \mathcal{R} \\
& \sum_{i \in \mathcal{P}^{0}} \sum_{j \in \mathcal{P}^{0}} \frac{f_{r} x_{i j r m} t_{i j m}}{365} \leq n_{r m} \quad \forall r \in \mathcal{R}, m \in \mathcal{M} \\
& \sum_{j \in \mathcal{P}^{0}} \sum_{r \in \mathcal{R}} \sum_{m \in \mathcal{M}} \frac{d_{i}}{x_{i j r m} f_{r}}(1+B) \leq y_{i} \quad \forall i \in \mathcal{P} \\
& f_{r} \in \mathbb{R}^{\geq 0} \quad \forall r \in \mathcal{R}, m \in \mathcal{M} \\
& n_{r m} \in \mathbb{Z}^{+} \quad \forall r \in \mathcal{R}, m \in \mathcal{M} \\
& x_{i j r m} \in\{0,1\} \quad \forall i \in \mathcal{P}^{0}, j \in \mathcal{P}^{0}, r \in \mathcal{R}, m \in \mathcal{M} \\
& y_{i} \in \mathbb{R}^{\geq 0} \quad \forall i \in \mathcal{P}
\end{aligned}
$$

The objective function (1) minimizes total annual cost for both chartering and operating the LNG tankers and for building and running the LNG infrastructure at the ports of demand. Constraints (2) require each port of demand to be visited by exactly one rotation. The set of constraints (3)-(5) defines the tanker routes. Constraints (3) and (4) are flow conservation and subtour elimination constraints, respectively. Constraints (5) state that each route can leave the supply port at most once. Routes, for which the left-hand side of its corresponding constraint is zero, represent empty routes that are not used. The frequency $f_{r}$ of each route is determined by constraints (6). The left-hand side equals the total demand served on route $r$ divided by the tanker capacity used on that route. The integer number of LNG tankers required on each route is determined by constraints (7). The left-hand side represents the absolute number of days of operational time required to serve demand on route $r$ with a single tanker, divided by the available number of days per year. Constraints (8) determine the necessary onshore infrastructure capacity at each port $i$. It is required to be at least as large as the amount of LNG received at each tanker visit plus a relative buffer capacity B. Finally, constraints (9)-(12) define the domain of the decision variables.

In the above formulation the amount of LNG unloaded at a port depends on the route. It equals the tanker capacity times the port's share of demand compared to the demand of the other ports on the same route. Hence, the model allows for partially loaded LNG tankers. As 
discussed above we furthermore assume a steady demand for LNG over time and thus periodic LNG deliveries. The maximum amount of LNG on stock at a location thus never exceeds the amount of LNG unloaded at each delivery. Fig. 4 illustrates the stock level at a single port that is the only port on a route on which two LNG tankers operate.

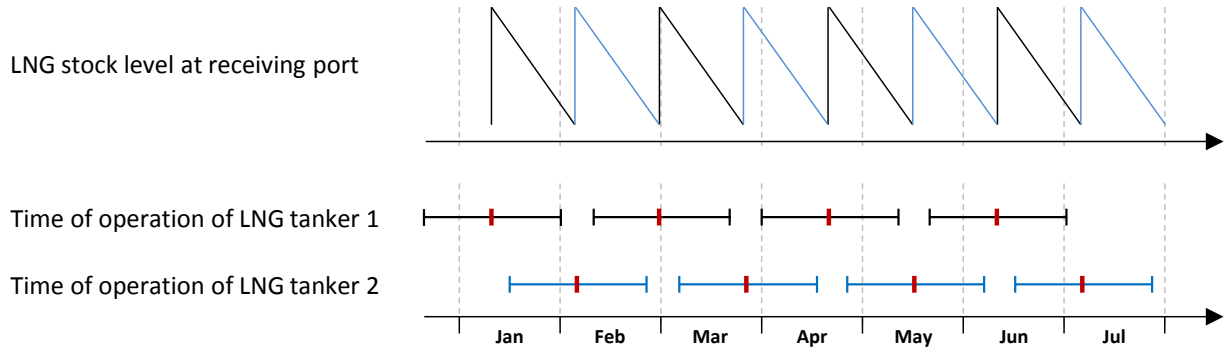

Figure 4: Illustration of the LNG stock level at a port that is supplied by a tanker service consisting of two tankers operating in parallel. The bars for each tanker illustrate their time of operation, with the red marks representing the time of delivery at the port.

The arc-based formulation is non-linear in its objective function and also in some of its constraints. It can be linearized at the cost of a larger set and number of decision variables and constraints. The problem quickly grows to intractable size due to the number of decision variables and constraints that exponentially depend on the number of ports of demand. In the next section we thus present a path-based formulation that allows to easily incorporate non-linearities.

\subsection{Path-based model formulation}

In the arc-based formulation we can observe the following: once the routes and the corresponding tanker types are defined, i.e. once the $x_{i j r m}$ variables are fixed, the values of the remaining decision variables follow directly from equations (6)-(8). Fixing the $x_{i j r m}$ variables corresponds to defining a set of routes and the tanker type used on each of them. In other words, once a route and the tanker type used on that route are decided, the route frequency, the number of tankers and the infrastructure capacity at the ports along the route can be deduced. Let $\mathcal{S}$ denote the set of all route-tanker type combinations $s$. We will call a route-tanker type combination $s$ with the associated route frequency $f_{s}$, number of LNG tankers $n_{s}$, LNG tanker capacity $q_{s}$ and the port infrastructure capacities $\left(y_{1}, \ldots, y_{|\mathcal{P}|}\right)_{s}$ a partial solution. With $f_{s}, n_{s}, q_{s}$ and $\left(y_{1}, \ldots, y_{|\mathcal{P}|}\right)_{s}$ being parameters of a partial solution, we can hence calculate the corresponding cost of each partial solution.

Example: Let us consider a partial solution for the route Qatar-Singapore-Shanghai-Qatar and a Q-max tanker type of $265000 \mathrm{~m}^{3}$ capacity as an example. The demand in Shanghai equals 12 times the demand in Singapore and therefore on each service 1/13 of the tanker's capacity $\left(\approx 20400 \mathrm{~m}^{3}\right)$ is unloaded in Singapore and $12 / 13\left(\approx 244600 \mathrm{~m}^{3}\right)$ are unloaded in Shanghai on each tanker round trip. The required onshore storage capacity at each location is calculated as the absolute amount of LNG unloaded at each delivery plus some buffer capacity, i.e. the required infrastructure capacity is much smaller in Singapore than in Shanghai. If the total annual demand for LNG at both ports is $11000000 \mathrm{~m}^{3}$, approximately 41.5 tanker loads are required throughout the whole year. As a single service - starting in Qatar, delivering the LNG to Singapore and Shanghai and returning to Qatar - takes around 35 days, 4 tankers are required to do 41.5 round trips during a single year (365 days).

If all partial solutions were given, we could solve the problem by optimally combining these partial solutions such that each port is covered by exactly one partial solution. This translates 
into a pure set-partitioning model with a set $\mathcal{S}$ of partial solutions defined by a route-tanker type pair $s=(r, m)$ with $r \in \tilde{\mathcal{R}}$ and $m \in \mathcal{M}$, and a set $\mathcal{P}$ of ports of demand. Note that we use $\tilde{\mathcal{R}}$ to denote the set of all possible routes, which should not be confused with the set $\mathcal{R}$ used in the arc-based formulation. Let $\hat{c}_{s}$ be the cost of partial solution $s \in \mathcal{S}$ and let $a_{i s}$ denote whether port $i \in \mathcal{P}$ is covered by partial solution $s\left(a_{i s}=1\right)$ or not $\left(a_{i s}=0\right)$. The resulting set-partitioning model is

$$
\begin{gathered}
\min \sum_{s \in \mathcal{S}} \hat{c}_{s} x_{s} \\
\text { s.t. } \sum_{s \in \mathcal{S}} a_{i s} x_{s}=1 \quad \forall i \in \mathcal{P} \\
x_{s} \in\{0,1\} \quad \forall s \in \mathcal{S}
\end{gathered}
$$

The partial solutions can either be enumerated in advance or, if the number of possible partial solutions is too large, the LP-relaxation of model (13)-(15) can be solved by generating partial solutions on the fly by using a delayed column generation algorithm. Note that almost any type of cost function can be used to define the cost $\hat{c}_{s}$ of a partial solution. The path-based formulation thus allows to include non-linearities in the cost while still solving a linear master problem. In Section 4.2 we provide a detailed description of the cost components used to define the cost of a partial solution $\hat{c}_{s}$ for the problem instance studied.

\subsection{Enumeration and pre-selection of partial solutions}

The number of partial solutions is of size $|\tilde{\mathcal{R}}| \times|\mathcal{M}|$. Since the size of set $\tilde{\mathcal{R}}$ depends exponentially on the number of ports, $|\mathcal{P}|$, so does the set of partial solutions $\mathcal{S}$. Based on the following observations, however, we can reduce the set $\mathcal{S}$ of partial solutions significantly: for each subset of ports, different routes exist and different tanker types can be used on each of these routes. Each pair of a route and a tanker type represents a partial solution. For a given set of ports, there will be at least one route-tanker type pair that dominates the others in terms of cost. For each unique subset of ports we hence only need to include one partial solution, namely the one that dominates all other partial solutions that cover the same subset of ports. By simple cost comparison we can thus eliminate a large part of the partial solutions in advance. The set of partial solutions that finally needs to be included in the set-partitioning model (13)-(15) is of size $\left|2^{|\mathcal{P}|}-1\right|$ and equal to the number of subsets of ports.

\section{Computational Results}

In this section we first provide a description of the case study and its underlying data, and a detailed description of the cost functions used in the path-based model (sec. 4.1 and 4.2). The results rely on a set of assumptions made by the company. In Section 4.3 we present selected scenarios that reflect different assumptions and allow to assess their impact. In Section 4.4 results for the different scenarios are presented and discussed. As some of the input parameters are subject to large fluctuations over time, we present further results based on a sensitivity analysis in Section 4.5.

\subsection{Case study}

Eight locations along the route Asia-Europe (see Fig. 3) had been preselected by the company as potential future points of supply of LNG for their container vessel fleet as well as for spot market demand. We were further provided with LNG demand estimates for each of these 


\begin{tabular}{lrr}
\hline Location & Demand $\left[\mathrm{m}^{3}\right]$ & $\begin{array}{r}\text { Demand as factor } \\
\text { of Malta demand }\end{array}$ \\
\hline Rotterdam & $4893.6 \mathrm{~K}$ & $46 \times$ \\
Algeciras & $851.1 \mathrm{~K}$ & $8 \times$ \\
Malta & $106.4 \mathrm{~K}$ & $1 \times$ \\
Port Said & $212.8 \mathrm{~K}$ & $2 \times$ \\
Salalah & $638.3 \mathrm{~K}$ & $6 \times$ \\
Jebel Ali & $638.3 \mathrm{~K}$ & $6 \times$ \\
Singapore & $851.1 \mathrm{~K}$ & $8 \times$ \\
Shanghai & $10212.8 \mathrm{~K}$ & $96 \times$ \\
\hline Total & $18404.3 \mathrm{~K}$ & $173 \times$ \\
\hline
\end{tabular}

Table 1: Locations and corresponding LNG demand estimates

ports. The data is summarized in Table 1. The ports of Rotterdam and Shanghai represent the largest points of demand. Both ports are strategic hubs for container shipping - Rotterdam in Europe and Shanghai in Asia - and therefore the most frequented ones for container vessels. However, only a part of the demand at these two ports is dedicated to fueling container ships. A significant part is meant for satisfying demand on the growing spot market. We note that the figures represent expected future demand, not the the current demand for LNG.

\subsection{Cost functions}

The annual cost $\hat{c}_{s}$ of a partial solution in the path-based formulation is composed of different cost factors (of which some have already been introduced in Section 3.1). Note that all individual cost factors that are introduced below correspond to partial solutions of the path-based formulation and may hence differ from their definition in the arc-based formulation. To distinguish the cost factors that relate to partial solutions in the path-based formulation from the ones in the arcbased formulation, we use the notation $\hat{c}$. All cost components are presented as annual costs in thousand US dollars $(\mathrm{k} \$)$ if not stated otherwise.

The charter cost of a partial solution $s$, denoted by $\hat{c}_{s}^{\text {ch }}$, represents an annual charter cost for the number of LNG tankers of capacity $q_{s}$ needed to serve annual demand on the corresponding route. As an estimate for the daily charter rate of a tanker with capacity $q_{s}$ in thousand US dollars we use the function $9.0616 \cdot q_{s}^{0.4492}$ that is based on empirical data for tankers and their corresponding charter rates. The charter rate function is shown in Figure 5a. The annual charter cost of a partial solution $s \in \mathcal{S}$ depends on the tanker capacity $q_{s}$ in thousand cubic meters and the number $n_{s}$ of tankers needed on the route,

$$
\hat{c}_{s}^{\mathrm{ch}}=365 \cdot\left(9.0616 \cdot q_{s}^{0.4492}\right) \cdot n_{s}
$$

The fuel cost has been estimated in a similar fashion, based on empirical data for LNG tankers that run on heavy fuel oil. Note that although the new regulations regarding sulphur emissions will also apply to LNG tankers, our fuel cost estimates are based on HFO fuelled LNG tankers as an approximation due to the lack of available data. We will, however, take possible differences in fuel cost under the new regulation into account by defining different cost scenarios. We distinguish between the consumption of HFO, which is used to fuel the tankers while sailing, and MGO, which is used during port stays while loading or unloading LNG. The respective fuel cost functions for partial solution $s \in \mathcal{S}$ are defined as

$$
\begin{aligned}
\hat{c}_{s}^{\mathrm{HFO}} & =\left(10.4293 \cdot q_{s}^{0.5028}\right) \cdot t_{s}^{\text {sail }} \cdot p^{\mathrm{HFO}} \\
\hat{c}_{s}^{\mathrm{MGO}} & =\left(2.9500 \cdot q_{s}^{0.5652}\right) \cdot t_{s}^{\mathrm{port}} \cdot p^{\mathrm{MGO}}
\end{aligned}
$$




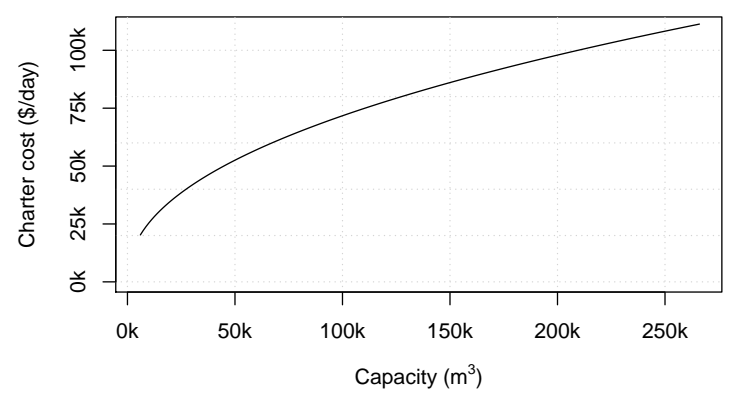

(a) Charter cost function

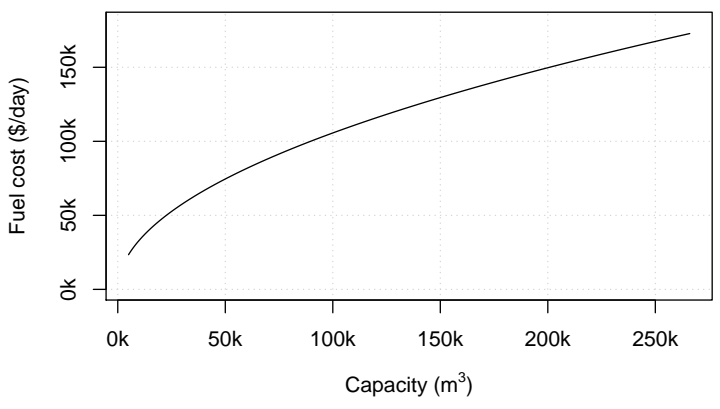

(b) Fuel cost function (HFO, sailing only)

Figure 5: Daily charter and fuel cost functions based on the tanker capacity

The fuel cost $\hat{c}_{s}^{\mathrm{HFO}}$ equals the tanker type dependant fuel consumption per day multiplied with the accumulated annual sailing time of this partial solution, $t_{s}^{\text {sail }}$, and is further multiplied with the fuel price per ton, $p^{\mathrm{HFO}}$. The parameter $t_{s}^{\text {sail }}$ is obtained through multiplication of the duration of a single route times the frequency of the route, $f_{s}$. The daily fuel consumption while sailing is based on an assumed speed of 18 knots. Figure 5b shows the daily fuel cost depending on the tanker capacity. The cost function $\hat{c}_{s}^{\mathrm{MGO}}$ for burning MGO during port stays represents the tanker dependant in-port fuel consumption per hour multiplied with the total in-port time, $t_{s}^{\text {port }}$, and multiplied with the fuel price per ton, $p^{\mathrm{MGO}}$. Bunker prices of $\$ 300$ and $\$ 450$ for HFO and MGO, respectively, are assumed in the base case of the study. The total fuel cost of a partial solution $s$ hence is $\hat{c}_{s}^{\text {fuel }}=\hat{c}_{s}^{\mathrm{HFO}}+\hat{c}_{s}^{\mathrm{MGO}}$. In practice the fuel cost function may further depend on the ship type, the ship's load and many other factors (see e.g. Psaraftis and Kontovas, 2014, for a discussion of fuel cost estimation). The path-based modelling framework allows to incorporate basically any type of fuel cost function, if the necessary information is available.

The annual infrastructure cost consists of capital expenditures (CAPEX) and operational expenditures (OPEX). We will consider the same, capacity-dependant investment cost at all ports. Even thoug investment options and cost may differ between different ports, the same cost function is used for all ports due to the lack of reliable data for each individual port. In order to estimate the capital expenditures for LNG terminals of different capacities, we use a capacity factor based estimator (see e.g. Westney, 1997). It uses historical records to estimate the cost of similar projects. The formula as shown below further assumes a non-linear increase in cost with increasing capacity, reflecting economies of scale (Fig. 6). The parameters $\bar{y}_{1}, \bar{y}_{2}$ and $\operatorname{CAPEX}\left(\bar{y}_{1}\right), \operatorname{CAPEX}\left(\bar{y}_{2}\right)$ represent capacities and respective capital expenditures of reference projects:

$$
\frac{\operatorname{CAPEX}\left(\bar{y}_{2}\right)}{\operatorname{CAPEX}\left(\bar{y}_{1}\right)}=\left(\frac{\bar{y}_{2}}{\bar{y}_{1}}\right)^{\delta}
$$

The parameter $\delta$, also called capacity factor, is estimated based on two anchor points provided by the company. They correspond to two existing LNG terminals of capacities $28500 \mathrm{~m}^{3}$ and $114000 \mathrm{~m}^{3}$, and CAPEX of $\$ 94 \mathrm{M}$ and $\$ 164 \mathrm{M}$, respectively. The resulting capacity factor used in our study is $\delta=0.4015$. The CAPEX of any LNG terminal with capacity $y$ can thus be calculated using the formula:

$$
\operatorname{CAPEX}(y)=\operatorname{CAPEX}\left(\bar{y}_{1}\right) \cdot\left(\frac{y}{\bar{y}_{1}}\right)^{0.4015}
$$

Equivalent to the arc-based model formulation, we require the capacity of an LNG terminal at port $i$ to be as large as the amount of LNG that is received with each tanker visit plus a buffer. 
For a given partial solution, the amount delivered at a port corresponds to its share of demand on the route multiplied with the capacity of the tanker used. Hence, the storage capacity $y_{i s}$ of a port $i$ of a partial solution $s$ can be determined as

$$
y_{i s}=\frac{a_{i s} d_{i}}{\sum_{i \in \mathcal{P}} a_{i s} d_{i}} q_{s}(1+B)
$$

The annual OPEX of an LNG terminal are estimated to lie at around $5 \%$ of the CAPEX. Let $t^{\text {life }}$ be the expected lifetime of the terminal. The total annual infrastructure cost of a partial solution is then calculated as the sum of the capital expenditures per year and the annual operational expenditures, i.e.

$$
\hat{c}_{s}^{\mathrm{inf}}=\sum_{i \in \mathcal{P}}\left(\operatorname{CAPEX}\left(y_{i s}\right) / t^{\mathrm{life}}+0.05 \cdot \operatorname{CAPEX}\left(y_{i s}\right)\right)
$$

In the study we assume a lifetime of 30 years for the LNG terminals.

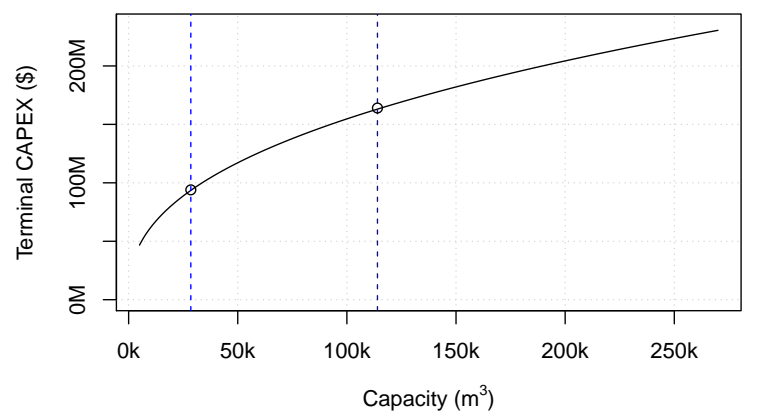

Figure 6: Terminal capital expenditures depending on the storage capacity. The two reference points used to calculate the capacity factor exponent $\delta$ are highlighted.

The port call cost is the cost charged by a port each time an LNG carrier berths and it is assumed to be equal at all ports considered. The cost factor $\hat{c}_{s}^{\text {port }}\left(q_{s}\right)$ represents the annual port call cost of a partial solution and depends on the carrier capacity $q_{s}$, the number of ports that are covered by the corresponding partial solution, $\left|\mathcal{P}_{s}\right|=\sum_{p \in \mathcal{P}} a_{p s}$, and the frequency of the service, $f_{s}$. The cost per visit depends on the tanker's capacity, which is divided into three capacity classes and corresponding port call fees. The port call cost of a solution in thousand US dollars is defined as

$$
\hat{c}_{s}^{\text {port }}= \begin{cases}60 \cdot\left|\mathcal{P}_{s}\right| \cdot f_{s} & \text { if } q_{s}<50 \\ 150 \cdot\left|\mathcal{P}_{s}\right| \cdot f_{s} & \text { if } 50 \leq q_{s}<120 \\ 300 \cdot\left|\mathcal{P}_{s}\right| \cdot f_{s} & \text { if } 120 \leq q_{s}\end{cases}
$$

Canal transit costs occur whenever a ship uses a canal. In our study the Suez canal is the only relevant canal. We use a function that linearly depends on the capacity of the tanker in order to approximate a single transit through the canal. For the smallest tanker considered $\left(5000 \mathrm{~m}^{3}\right)$ we assume a cost of $\$ 100 \mathrm{k}$ per transit and for the largest carrier considered $\left(265000 \mathrm{~m}^{3}\right)$ a cost of $\$ 500 \mathrm{k}$ per transit. Canal transit costs for tankers with a capacity in between are obtained through linear interpolation. The annual canal transit costs of a partial solution are thus calculated as

$$
\hat{c}_{s}^{\text {canal }}=2 \cdot f_{s} \cdot\left(100+\frac{q_{s}-5}{265-5} \cdot(500-100)\right)
$$


In addition to the cost factors included in the arc-based problem formulation, in the pathbased formulation we further consider inventory cost for LNG on board the tankers during transport and at the destination ports before consumption. With a partial solution given, we can calculate the average amount of LNG on tankers and in onshore storage tanks throughout a year. Let $\bar{l}_{s}$ denote the average inventory of LNG in thousand cubic meters of partial solution $s$ throughout a year. Assuming a value of $\$ 220 / \mathrm{m}^{3}$ of LNG $(\approx \$ 9.16 / \mathrm{mmBTU}$ of LNG $)$ and an inventory cost rate of $6 \%$ per year in our study, the annual inventory cost of a partial solution in thousand US dollars is defined by

$$
\hat{c}_{s}^{\text {inv }}=\bar{l}_{s} \cdot 220.00 \cdot 1.06
$$

Any of these cost functions can be adapted to different settings. Although we neglect the loss of LNG due to boil-off in our study, it can also be accounted for in path-based formulations (see e.g. Grønhaug et al., 2010). Finally, the total cost of a partial solution is given by the sum of all cost factors,

$$
\hat{c}_{s}=\hat{c}_{s}^{\mathrm{ch}}+\hat{c}_{s}^{\mathrm{fuel}}+\hat{c}_{s}^{\mathrm{inf}}+\hat{c}_{s}^{\text {port }}+\hat{c}_{s}^{\mathrm{canal}}+\hat{c}_{s}^{\text {inv }}
$$

We can calculate the total cost of any partial solution using equations (16)-(26) and, as described above in Section 3.3, eliminate many partial solutions by simple cost comparison, as for each subset of ports one partial solution will dominate all the other partial solutions for that same subset of ports.

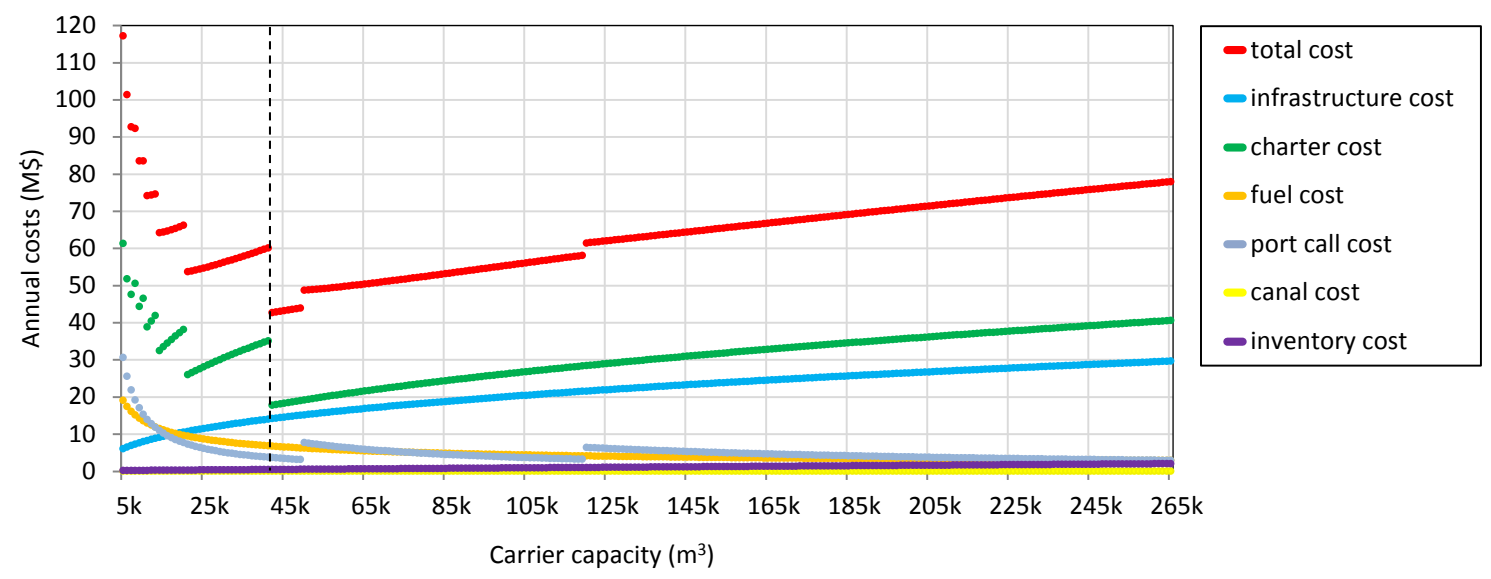

Figure 7: Costs of different partial solutions for the route Qatar-Jebel Ali-Salalah-Qatar. For the given route, the tanker capacity of $42000 \mathrm{~m}^{3}$ dominates all other tanker capacities in terms of total cost.

Figure 7 illustrates costs of different partial solutions for the route Qatar-Jebel Ali-SalalahQatar, with each of these partial solutions corresponding to a different tanker capacity in the range $5000-265000 \mathrm{~m}^{3}$. The partial solution with the lowest cost is denoted by the dashed line. All costs represent annual costs for satisfying the demand at the two locations. The charter cost is increasing with the tanker capacity and the steps result from different numbers of tankers needed to satisfy demand. For tanker capacities of $21000-41000 \mathrm{~m}^{3}$, for example, two tankers are necessary to satisfy demand at the two locations. Two tankers of $21000 \mathrm{~m}^{3}$ would have a much higher utilization than two tankers of $41000 \mathrm{~m}^{3}$, though. With a tanker capacity of $42000 \mathrm{~m}^{3}$ and higher, only a single tanker is necessary to satisfy annual demand. The fuel cost is decreasing with increasing tanker capacities, as larger tankers are more fuel efficient given the same total amount of LNG being transported (see Figure 5b). The port call costs are different for the three capacity classes of tankers (see the port call cost function (23)), and within each class decreasing with increasing tanker capacities, as larger capacities imply a 
lower number of deliveries of LNG. Note that for the location subset \{Jebel Ali, Salalah\} one alternative route exists (Qatar-Salalah-Jebel Ali-Qatar) whose corresponding partial solutions are not represented in this graph. Only the lowest-cost route-tanker type combination of the location subset $\{$ Jebel Ali, Salalah\} would finally be included in the set-partitioning problem.

\subsection{Scenarios}

The cost functions presented above are just one way of defining different types of cost and we will refer to the above described scenario as scenario $\mathbf{A}$. The simple structure of the path-based problem formulation allows for any type of cost functions that depend on the route and tanker type of a partial solution. We thus present two other scenarios that differ from scenario $A$. The two scenarios are not exhaustive, of course, but represent examples of changing underlying decisions and corresponding assumptions and how they can be evaluated using the model.

Scenario B In scenario $A$, the onshore storage capacities are chosen such that they exactly meet the requirements imposed by the chosen service. That is, the capacities equal just the amount of LNG that is unloaded at each tanker visit, plus some buffer capacity. The assumption tends to underestimate infrastructure cost and selects onshore capacities that are fully utilized under the given demand scenario and thus might not be robust to changes in the input. In scenario $B$ we require the onshore capacity to be at least as large as the capacity of the tanker type that serves the port, independent of the amount unloaded. That is, equation (21) of scenario A is replaced by

$$
y_{i s}=a_{i s} q_{s}(1+B)
$$

The infrastructure cost of the solution again is defined by equation (22), but with different infrastructure capacities as input. Under this assumption the onshore infrastructure cost will rather be overestimated. However, an advantage of any solution obtained under scenario $B$ is that any solution of partial tanker loads can be transformed into a solution in which tankers are required to be either full or empty (not partially loaded). A solution hence provides an upper bound on the cost for the case in which partial tanker loads are not feasible. If a solution, for example, covers two ports with the tanker unloading half its load at each port, it can be transformed into a solution where the tanker covers only one port per trip, but serves the ports alternately, serving the same port on every second trip. Scenario $B$ is interesting from an industrial point of view because the majority of LNG tankers currently under construction are of the membrane type. These are known to suffer from sloshing and consequently rather strict filling levels apply to these tankers (Delorme et al., 2005).

Scenario C In a third scenario, scenario $C$, we assume that tanker charter costs only apply for the actual time of utilization, not for the whole year. Hence, no cost is assumed for tankers while not being used and the original charter cost function (16) is replaced by the modified charter cost function

$$
\hat{c}_{s}^{\mathrm{ch}}=365 \cdot u_{s} \cdot\left(9.0616 \cdot q_{s}^{0.4492}\right) \cdot n_{s}
$$

with $u_{s}$ denoting the tanker utilization of the corresponding partial solution $s$. In this scenario the obtained charter cost can be interpreted as a lower bound on the expected charter cost.

\subsection{Results}

We implemented an algorithm for efficiently enumerating all partial solutions, which constitute the input to the set partitioning model. The set-partitioning models were solved using CPLEX Optimization Studio. The tests were executed on a machine with an Intel Core i7-4600U CPU 


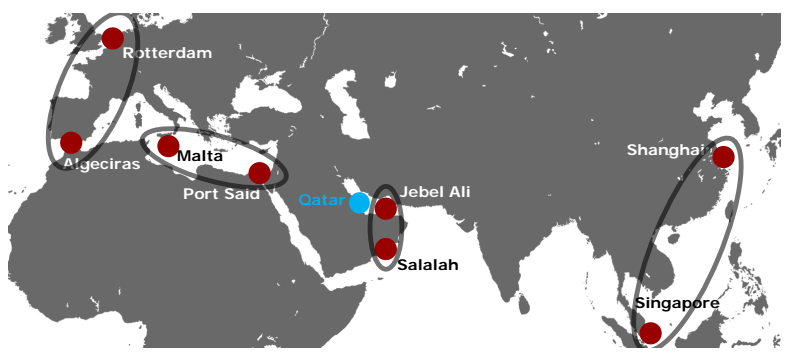

(a) Scenario A

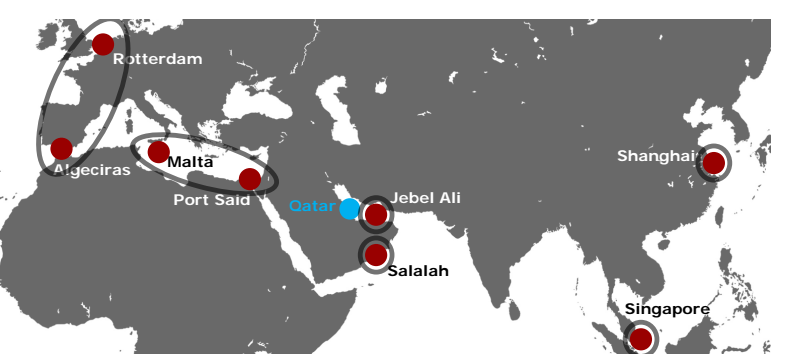

(b) Scenario B

Figure 8: Optimal clustering of ports. All ports within a cluster are served on round trips and by the same tanker type.

with $2.1 \mathrm{GHz}$. The generation of the partial solutions takes around 30 seconds on average and the solution time of the set-partitioning model is less than a second.

The results for scenario A are summarized in Table 2. In the cost optimal solution all ports are clustered pairwise, as illustrated in Figure 8a. The charter costs dominate with almost making up half of the total costs, followed by fuel costs (21\%) and infrastructure costs $(14 \%)$. The share of the different cost factors, however, differs substantially between the various partial solutions. The partial solutions covering the ports of Shanghai and Rotterdam, which represent more than $80 \%$ of the total demand for LNG, also constitute more than $85 \%$ of the total cost. Due to Shanghai's and Rotterdam's large distances from Qatar, charter and fuel cost per transported unit of LNG are very high compared to the other cost components (50\% and $22 \%$, resp.). In the partial solution that covers demand in Malta and Port Said, the share of charter and fuel cost $(36 \%$ and $14 \%$, resp.) is significantly lower, while infrastructure cost and also canal transit cost have a much larger weight (30\% and 13\%, resp.). We further observe that for each cluster of ports the optimal tanker capacity corresponds to the capacity that minimizes the necessary number of LNG tankers but maximizes their utilization. The optimal infrastructure capacity per port is given in Table 5 .

There are several trade-offs inherent in any solution. Clustering ports and assigning them to a single tanker round trip allows to use larger, more cost-efficient tankers while keeping their utilization high. In scenario A it further allows to reduce the cost for infrastructure, as only a fraction of the tanker load will be unloaded at each port visit. On the other hand, with more ports combined on a route the total sailing distance and the total number of port visits and canal transits (where required) are larger compared to serving each port individually, given the same tanker capacity.

Table 3 summarizes the results obtained for scenario B, where the infrastructure capacity is required to be as large as the capacity of the tanker that serves the corresponding port, inde-

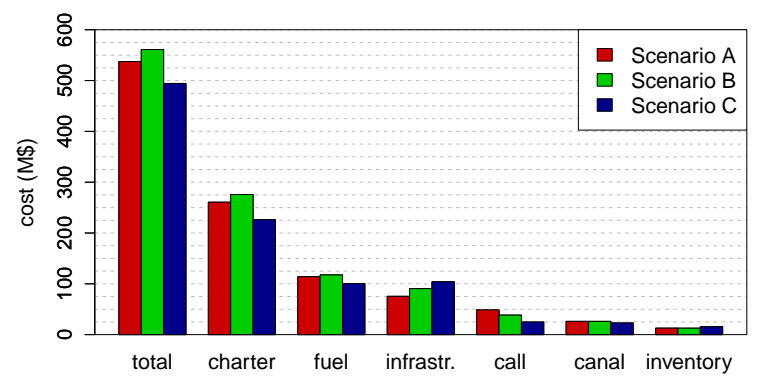

Figure 9: Comparison of cost of the optimal solution between the different scenarios. 


\begin{tabular}{|c|c|c|c|c|c|c|c|c|c|c|c|}
\hline \multirow[b]{2}{*}{ Route } & \multicolumn{4}{|c|}{ Characteristics } & \multicolumn{7}{|c|}{ Annual cost $(\mathrm{M} \$)$} \\
\hline & 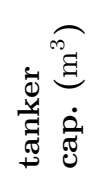 & 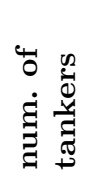 & 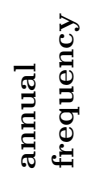 & 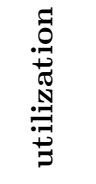 & 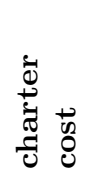 & 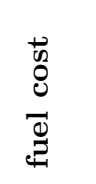 & 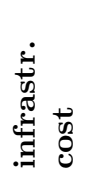 & 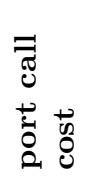 & 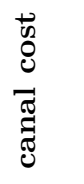 & 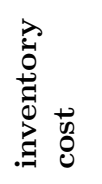 & 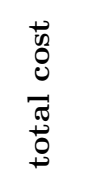 \\
\hline Algeciras - Rotterdam & $255 \mathrm{k}$ & 2 & 22.5 & $100 \%$ & 79.7 & 35.6 & 27.0 & 13.5 & 21.8 & 5.0 & 182.7 \\
\hline Port Said - Malta & $18 \mathrm{k}$ & 1 & 17.7 & $99 \%$ & 12.1 & 4.6 & 9.9 & 2.1 & 4.3 & 0.2 & 33.3 \\
\hline Jebel Ali - Salalah & $42 \mathrm{k}$ & 1 & 30.4 & $98 \%$ & 17.7 & 6.7 & 14.1 & 3.6 & 0.0 & 0.4 & 42.7 \\
\hline Singapore - Shanghai & $226 \mathrm{k}$ & 4 & 49.0 & $100 \%$ & 151.0 & 67.0 & 24.3 & 29.4 & 0.0 & 7.2 & 278.9 \\
\hline Total & & & & & 260.6 & 113.9 & 75.4 & 48.7 & 26.1 & 12.8 & 537.5 \\
\hline Total $(\%)$ & & & & & $48 \%$ & $21 \%$ & $14 \%$ & $9 \%$ & $5 \%$ & $2 \%$ & $100 \%$ \\
\hline
\end{tabular}

Table 2: Optimal solution to scenario A: Each row represents an optimal partial solution and its characteristics and costs. The first column indicates the route that is sailed in the corresponding partial solution, always starting and ending in Qatar. The following two columns denote the number and capacity of tankers used. The frequency denotes how often the round trip is sailed every year and equivalent to the number of port calls at each port along the respective route. The utilization indicates the relative time per year the tankers are in operation. The remaining columns denote the different annual cost per partial solution.

pendent of the amount of LNG being unloaded. Scenario B is more restrictive than scenario A and the total annual cost of its optimal solution is $4.4 \%$ higher than the cost of the corresponding optimal solution in scenario A. As could be expected, the total infrastructure capacity is significantly higher than in scenario A (see Table 5). The effect of the assumption introduced under scenario B, however, is dampened by the use of smaller tankers and a larger number of individually served ports compared to the optimal solution of scenario A. The ports of Shanghai, Singapore, Salalah and Jebel Ali are served on individual round trips whereas they are served pairwise in scenario A (see Figure 8b). Scenario B's additional requirement represents a reduced incentive to combine multiple ports on a round trip and a greater incentive to use LNG tankers of smaller capacity. Again, in almost all cases the capacities of the tankers are chosen such that the number of tankers is minimized and their utilization maximized. Overall, the optimal solution under scenario $B$ is $\$ 23.6 \mathrm{M}$ more expensive than the optimal solution under scenario $A$. In other words, if the problem of sloshing can be resolved and partial tanker loadings are feasible in the future, it may allow to reduce the total annual cost by more than $4 \%$.

\begin{tabular}{|c|c|c|c|c|c|c|c|c|c|c|c|}
\hline \multirow[b]{2}{*}{ Route } & \multicolumn{4}{|c|}{ Characteristics } & \multicolumn{7}{|c|}{ Annual cost $(M \$)$} \\
\hline & 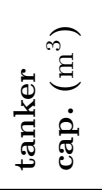 & 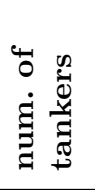 & 苂 & 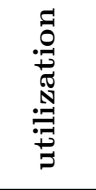 & 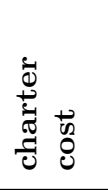 & $\begin{array}{l}\vec{n} \\
0 \\
0 \\
0 \\
0\end{array}$ & 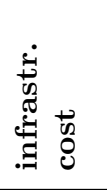 & 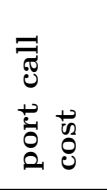 & 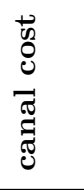 & 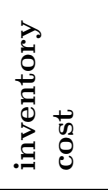 & 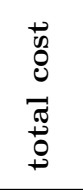 \\
\hline Algeciras - Rotterdam & $255 \mathrm{k}$ & 2 & 22.5 & $100 \%$ & 79.7 & 35.6 & 38.5 & 13.5 & 21.8 & 5.0 & 194.2 \\
\hline Port Said - Malta & $18 \mathrm{k}$ & 1 & 17.7 & $99 \%$ & 12.1 & 4.6 & 13.3 & 2.1 & 4.3 & 0.2 & 36.6 \\
\hline Salalah & $20 \mathrm{k}$ & 1 & 31.9 & $98 \%$ & 12.7 & 4.7 & 6.9 & 1.9 & 0.0 & 0.3 & 26.5 \\
\hline Jebel Ali & $8 \mathrm{k}$ & 1 & 79.8 & $53 \%$ & 8.4 & 1.2 & 4.8 & 4.8 & 0.0 & 0.1 & 19.2 \\
\hline Singapore & $43 \mathrm{k}$ & 1 & 19.8 & $99 \%$ & 17.9 & 7.2 & 9.4 & 1.2 & 0.0 & 0.6 & 36.3 \\
\hline Shanghai & $206 \mathrm{k}$ & 4 & 49.6 & $100 \%$ & 144.9 & 64.2 & 17.7 & 14.9 & 0.0 & 6.7 & 248.2 \\
\hline Total & & & & & 275.7 & 117.5 & 90.6 & 38.4 & 26.1 & 12.7 & 561.1 \\
\hline Total (\%) & & & & & $49 \%$ & $21 \%$ & $16 \%$ & $7 \%$ & $5 \%$ & $2 \%$ & $100 \%$ \\
\hline
\end{tabular}

Table 3: Optimal solution to scenario B: Each row represents an optimal partial solution and its characteristics and costs.

In scenario $\mathrm{C}$ charter costs are only incurred for the time tankers are in operation and not for 
the whole year, i.e. idle times are free of charge. A summary of the optimal solution for scenario $\mathrm{C}$ is given in Table 4. Port Said, Malta and Algeciras are served in a single LNG tanker round trip, while all other ports are served by individual round trips. The optimal solution appears to be in line with intuition: The average tanker size is significantly larger compared to scenario A, because the larger the tanker is, the lower are the charter and fuel costs per unit of LNG, and, most notably, utilization rates are much lower, as idle times are not penalized anymore. Due to the increased tanker capacities, the onshore infrastructure capacities are even larger than under scenario B (see Table 5). The more impacting charter costs are reduced at the price of a less heavy increase in annual infrastructure cost. Note that in scenario $\mathrm{C}$ the required infrastructure capacity at a port is, like in scenario A, a function of the amount unloaded, which represents an incentive to combine ports on round trips.

\begin{tabular}{|c|c|c|c|c|c|c|c|c|c|c|c|}
\hline \multirow[b]{2}{*}{ Route } & \multicolumn{4}{|c|}{ Characteristics } & \multicolumn{7}{|c|}{ Annual cost in million USD } \\
\hline & 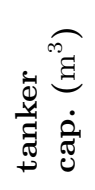 & 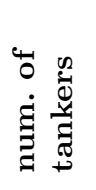 & 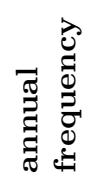 & 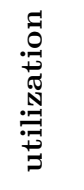 & 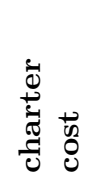 & $\begin{array}{l}\dot{n} \\
0 \\
0 \\
0 \\
0 \\
0\end{array}$ & 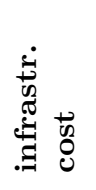 & 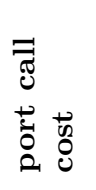 & $\begin{array}{l}\overrightarrow{0} \\
0 \\
0 \\
\tilde{0} \\
\tilde{0}\end{array}$ & 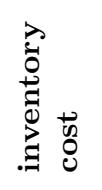 & $\begin{array}{l}0 \\
0 \\
0 \\
0 \\
\pi \\
0 \\
0 \\
0\end{array}$ \\
\hline Rotterdam & $265 \mathrm{k}$ & 2 & 18.5 & $81 \%$ & 65.4 & 29.5 & 19.6 & 5.5 & 18.5 & 4.6 & 143.1 \\
\hline Port Said - Malta - Algeciras & $242 \mathrm{k}$ & 1 & 4.8 & $35 \%$ & 13.6 & 6.0 & 33.3 & 4.4 & 4.5 & 2.2 & 63.9 \\
\hline Salalah & $49 \mathrm{k}$ & 1 & 13.0 & $41 \%$ & 7.7 & 3.1 & 9.9 & 0.8 & 0.0 & 0.5 & 21.9 \\
\hline Jebel Ali & $24 \mathrm{k}$ & 1 & 26.6 & $18 \%$ & 2.5 & 0.7 & 7.5 & 1.6 & 0.0 & 0.2 & 12.4 \\
\hline Singapore & $119 \mathrm{k}$ & 1 & 7.2 & $36 \%$ & 10.3 & 4.4 & 14.2 & 1.1 & 0.0 & 1.1 & 31.1 \\
\hline Shanghai & $265 \mathrm{k}$ & 4 & 38.5 & $78 \%$ & 126.7 & 57.0 & 19.6 & 11.6 & 0.0 & 7.1 & 221.9 \\
\hline Total & & & & & 226.2 & 100.6 & 104.0 & 24.9 & 23.0 & 15.6 & 494.3 \\
\hline Total (\%) & & & & & $46 \%$ & $20 \%$ & $21 \%$ & $5 \%$ & $5 \%$ & $3 \%$ & $100 \%$ \\
\hline
\end{tabular}

Table 4: Optimal solution to scenario C: Each row represents an optimal partial solution and its characteristics and costs.

Depending on which scenario is considered, the optimal strategic decisions can be very different, as e.g. the optimal onshore storage tank capacities indicate (Table 5). The relative difference in cost between the corresponding optimal solutions, however, is still rather moderate (Fig. 9), especially against the background of considerable uncertainty in the input parameters. The latter will be addressed in the following section through a sensitivity analysis.

\begin{tabular}{lrrr}
\hline Port & Scenario A & Scenario B & Scenario C \\
\hline Rotterdam & 228.1 & 267.8 & 278.3 \\
Algeciras & 39.7 & 267.8 & 184.8 \\
Malta & 6.3 & 18.9 & 23.1 \\
Port Said & 12.6 & 18.9 & 46.2 \\
Salalah & 22.1 & 21 & 51.5 \\
Jebel Ali & 22.1 & 8.4 & 25.2 \\
Singapore & 18.2 & 45.2 & 125.0 \\
Shanghai & 218.1 & 216.3 & 278.3 \\
\hline Total & 567.0 & 864.2 & 1012.2 \\
Total vs. Scenario A & & $+52.4 \%$ & $+78.5 \%$ \\
\hline
\end{tabular}

Table 5: Optimal onshore infrastructure capacities in thousand $\mathrm{m}^{3}$ for all ports and under different scenarios. 


\subsection{Sensitivity Analysis}

Many of the input parameters are subject to heavy fluctuations over time and therefore extremely hard to predict. By early 2016, the heavy fuel oil price has, against the forecasts, plummeted to a value below $\$ 300$ per tonne since August 2014, after having been above $\$ 600$ for more than three years. Similarly, charter rates may fluctuate heavily over time. The average daily charter rate for 12 months time charters for a $160000 \mathrm{~m}^{3}$ LNG tanker, for example, has varied between $\$ 30000$ and $\$ 150000$ since 2010 (RS Platou Economic Research AS, 2015). For other input parameters as e.g. the infrastructure cost (as a function of capacity) even current estimates are generally provided as interval estimates due to many factors of uncertainty. In this section we show how the presented modelling approach can be used to test the robustness of the above solutions against fluctuations of the fuel price, charter rates and expected infrastructure costs compared to the base case. The sensitivity analysis was conducted for scenarios A and B exclusively due to their practical relevance. The focus of the sensitivity analysis lies on the strategic infrastructure sizing and tanker investment decisions, as the reversion or adjustment of strategic decisions is usually not possible without considerable extra costs.

We tested seven different charter rate levels including the base case. As the charter rate is a function of the tanker capacity, the changes in charter costs are provided as relative deviations from the base cost function in percent. Values in the range $-50 \%$ to $+100 \%$ of the base charter cost function have been evaluated, i.e. the lowest representing half and the highest representing twice the base case charter rate. To evaluate the solution robustness against fluctuations in the fuel price we tested five different equally distant values in the range of $\$ 150-\$ 750$ per tonne of HFO, with $\$ 300$ corresponding to the base case. As in the base case, the price of MGO was generally set to 1.5 times the price of HFO. We furthermore evaluated the impact of infrastructure cost changes in the range of $-50 \%$ to $+100 \%$ of the annual infrastructure cost function $(\mathrm{CAPEX}+\mathrm{OPEX})$ as defined in the base case. Note that each parameter variation was tested individually against the base case of the corresponding scenario.

The results of the sensitivity analysis are summarized in Figures 10 and 11. Figure 10 shows selected properties of each case's optimal solution and how it changes with varying input parameters. In the given study we are mainly interested in how robust irreversible decisions like the number and size of tankers and the size of the onshore infrastructure are. Generally, the impact of all tested parameter fluctuations on the average optimal onshore storage capacities is very limited under both scenarios, with the average (optimal) infrastructure capacity varying between $71000 \mathrm{~m}^{3}$ and $78000 \mathrm{~m}^{3}$. The largest observed relative change in capacity for a single port was $17 \%$. The optimal number of tankers and their capacity appears to be more sensitive to the parameter fluctuations, at least under scenario B. Under some parameter fluctuations the average number of tankers can vary by up to $33 \%$ and the average tanker capacity by up to $29 \%$ compared to the optimal solution of the base case.

Although the optimal solutions may look substantially different under some parameter realizations, the total cost savings compared to the optimal solution of the base case turn out to be relatively low. Figure 11 illustrates the relative changes in all cost components of an optimal solution compared to the base case. Figure 11e, for example, illustrates a case where the optimal solution of the base case remains optimal under all tested infrastructure cost variations in scenario A. This is not the case under scenario B, but Figure 11f shows that the adjustments of the optimal solution reduce the impact on some cost factors and increase the impact on others. Figure 12 shows the savings in total cost that could be achieved if reoptimization was possible after different parameter realizations. Interestingly it turns out that none of the reoptimized solutions reduces the total cost by more than $0.8 \%$ compared to the solution that is optimal for the base case. On the contrary, the solution to the base case remains optimal or very close to optimal under most of the tested parameter variations.

These results can be interpreted in two ways: the optimal solution to the base case appears to be quite robust to changes in the main cost functions, i.e. the optimal solution to the base 
case remains a very good solution under the evaluated parameter changes. On the other hand, however, it means that in practice there is very little leeway for the company to absorb cost increases in the investigated case study, even if major strategic decisions were reversible or adjustable in the future. These insights are very valuable, especially when it comes to risk assessment of large projects like the one considered.

\section{Conclusion}

The paper has introduced a novel, non-linear model for solving a strategic infrastructure sizing problem. The model decides the dimensions of a number of LNG depots that are meant to serve a fleet of LNG fuelled container ships as well as demands for LNG in the spot market. The model includes a routing component in order to account for transportation from the LNG source to the depots that are spread over Europe and Asia. The model was developed as part of a study (conducted by one of the major global container lines) that examined the feasibility of operating container ships by LNG. The non-linear model proves difficult to solve (Boleda Molas, 2015) and consequently an equivalent set partitioning formulation and necessary preprocessing techniques are proposed. The proposed model allows to solve realistic problem instances that have been presented in the paper within a very short time. The presented modelling framework is highly flexible and allows to incorporate almost any type of cost functions and underlying assumptions. Quick solution times allow decision makers to investigate different scenarios and better understand cause-and-effect relationships. The case study shows that the costs of transporting the LNG from its source to its destinations vastly exceed the cost of building and operating the necessary onshore infrastructure when considering a 30 year life time of the infrastructure. These results emphasize the necessity and advantages of optimizing infrastructure investment decisions and interdependent operational decisions simultaneously. Due to the volatility of the input parameters as well as due to the long planning horizon of the study, any optimal solution is associated with a large degree of uncertainty. Consequently a sensitivity analysis has been conducted. The results show that the obtained solutions are quite robust in terms of solution quality, but that any changes in individual cost factors will be almost fully reflected in the total cost. It is worth noting that the study was initiated in a time when oil prices where much higher compared to what they are at the time of writing. At this moment of time, however, it is not clear yet how the stricter sulphur regulations will affect marine gas oil and marine diesel oil prices once they are globally binding.

Several interesting extensions of the problem studied can be considered in future work. The large degree of stochasticity in the input data suggests the use of Stochastic Programming to deal with the uncertainty. A stochastic model could optimize over a larger set of scenarios and select solutions that are robust to fluctuations. Since the deterministic model is rather easy to solve it seems realistic, from a computational point of view, to make the move towards a stochastic model. Another possible extension is to broaden the decision problem and include the selection of ports that shall accommodate LNG terminals. This change would require to explicitly include the container ship routes in the model, since fewer refuelling options may imply that the container ships need larger LNG fuel tanks. There is a tradeoff between the LNG tank size on board of container ships and the vessels' container loading capacity. Thus, such a change would make the problem much more complex.

\section{Acknowledgements}

The research was supported by The Danish Strategical Research Council and The Danish Energy Technology Development and Demonstration Program (EUDP) under the ENERPLAN and GREENSHIP project. 
Number of routes

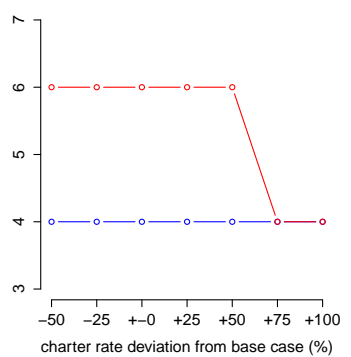

Avg. tanker capacity $\left(\mathrm{m}^{3}\right)$

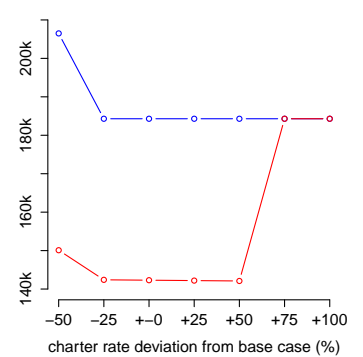

Number of tankers

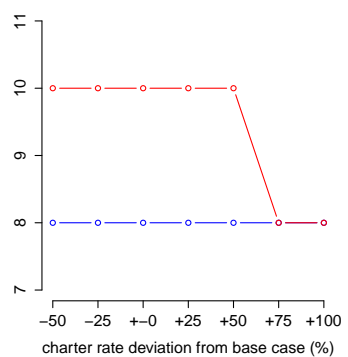

Avg. onshore storage size $\left(\mathrm{m}^{3}\right)$

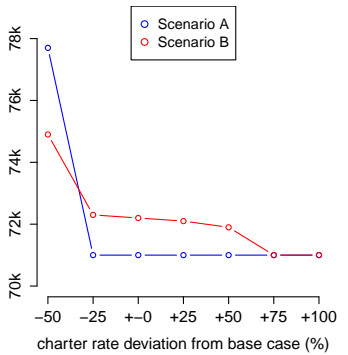

(a) Solution properties for scenarios A and B under varying charter rates. The $\mathrm{x}$-axis shows the \% deviation from the scenario's base charter rate function.

Number of routes

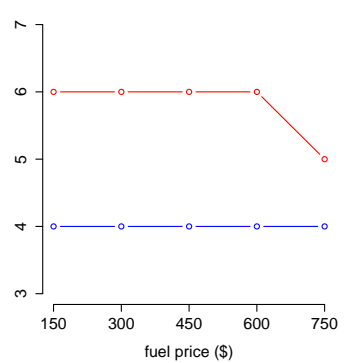

Avg. tanker capacity $\left(\mathrm{m}^{3}\right)$

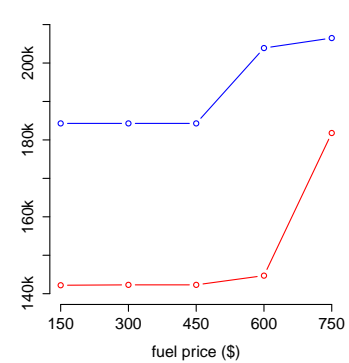

Number of tankers

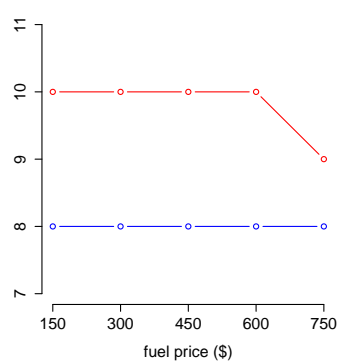

Avg. onshore storage size $\left(\mathrm{m}^{3}\right)$

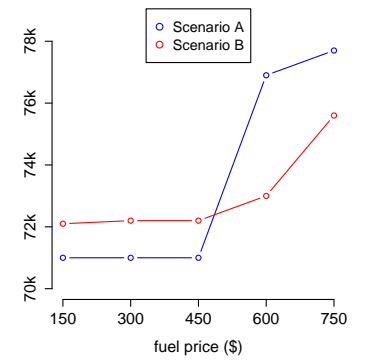

(b) Solution properties for scenarios A and B under varying fuel prices. The $\mathrm{x}$-axis shows the different tested fuel prices.

Number of routes

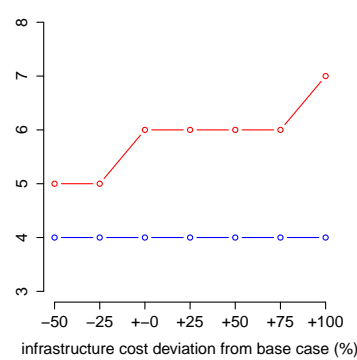

Avg. tanker capacity $\left(\mathrm{m}^{3}\right)$

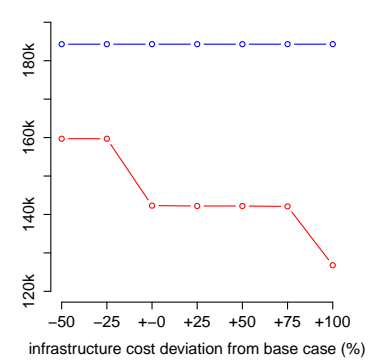

Number of tankers

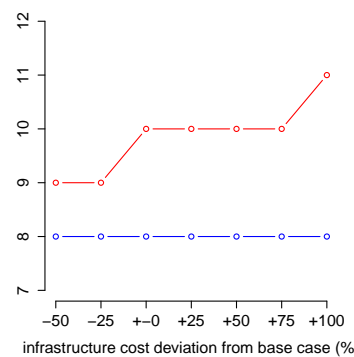

Avg. onshore storage size $\left(\mathrm{m}^{3}\right)$

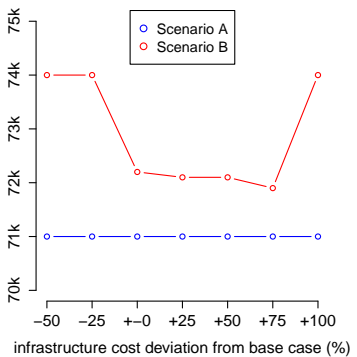

(c) Solution properties for scenarios A and B under varying infrastructure costs. The x-axis shows the $\%$ deviation from the scenario's base infrastructure cost function.

Figure 10: Properties of the optimal solution for different scenarios and parameters 


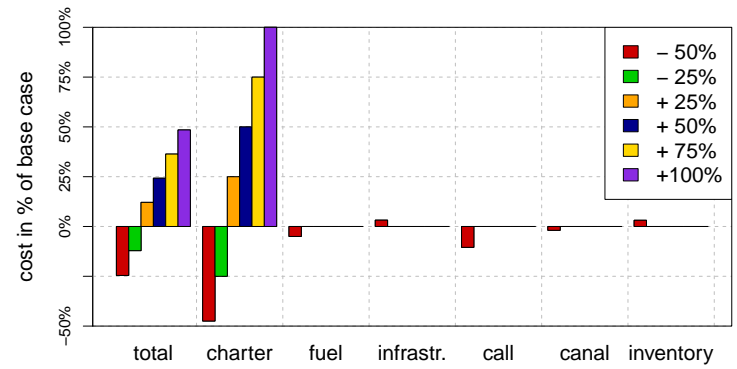

(a) Scenario A: Impact of fluctuations in the charter rates on the different cost factors

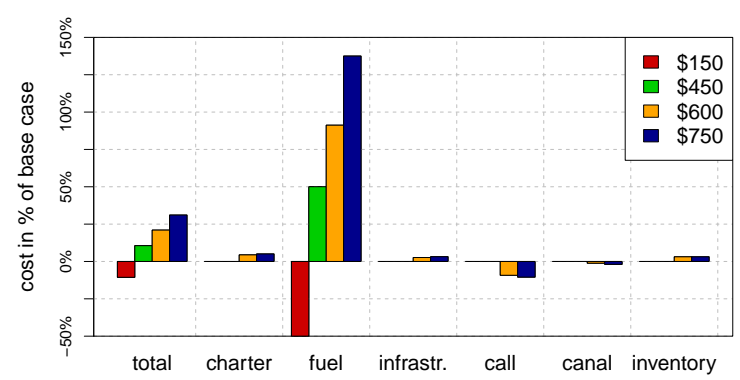

(c) Scenario A: Impact of fluctuations in the fuel (d) price (base: $\$ 300$ ) on the different cost factors

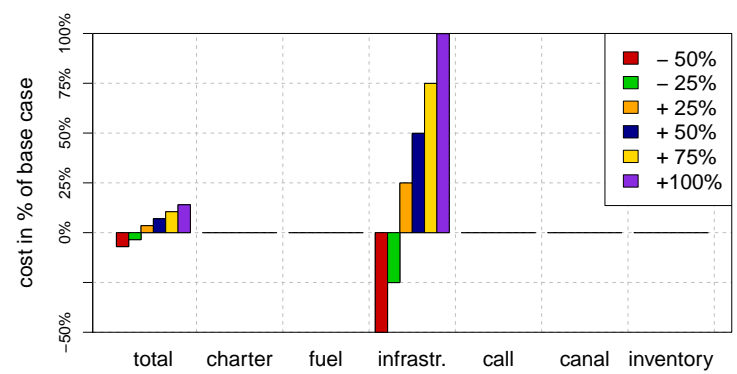

(e) Scenario A: Impact of fluctuations in the infrastructure cost on the different cost factors

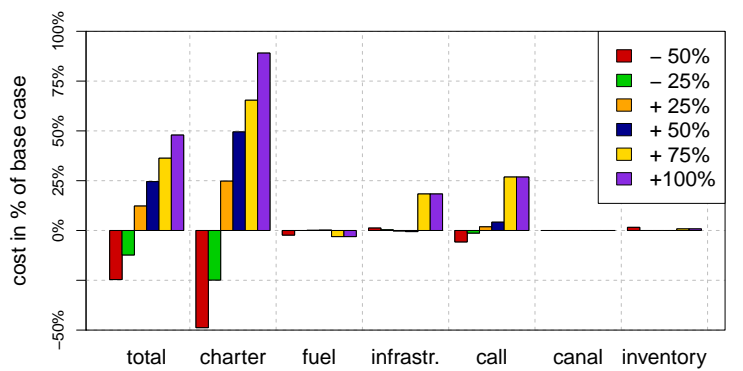

(b) Scenario B: Impact of fluctuations in the charter rates on the different cost factors

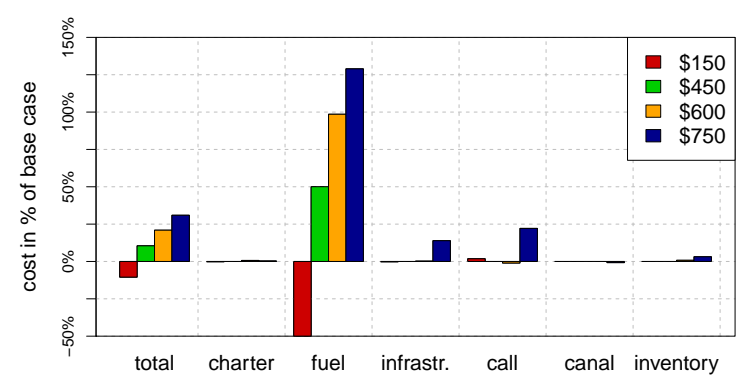

(d) Scenario B: Impact of fluctuations in the fuel price (base: $\$ 300$ ) on the different cost factors

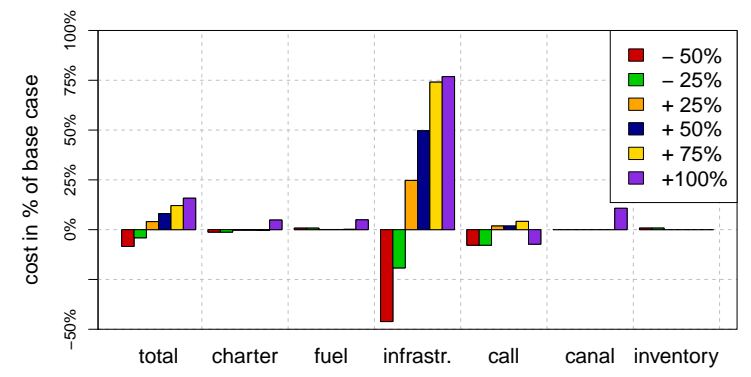

(f) Scenario B: Impact of fluctuations in the infrastructure cost on the different cost factors

Figure 11: Impact of varying input parameter values on cost factors of optimal solutions under scenario A and B. Each subfigure shows the relative change in different annual cost factors (total costs, charter costs, fuel costs, infrastructure costs, port call costs, canal transit costs, inventory costs) given the change of a selected input parameter (e.g. charter rate). All values represent relative changes compared to the costs in the optimal solution of the corresponding scenario's base case. 


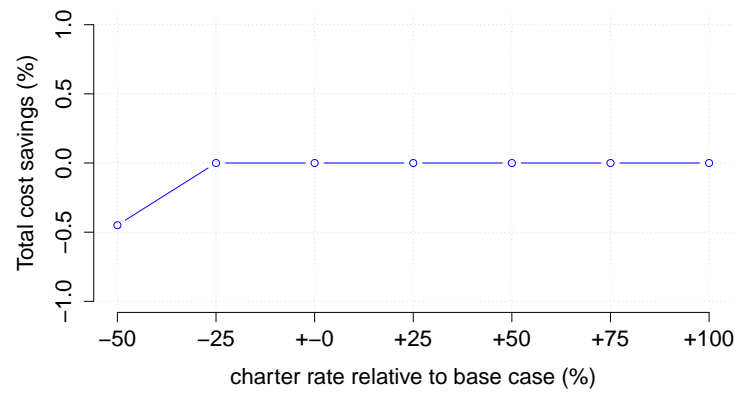

(a) Scenario A, varying charter rates

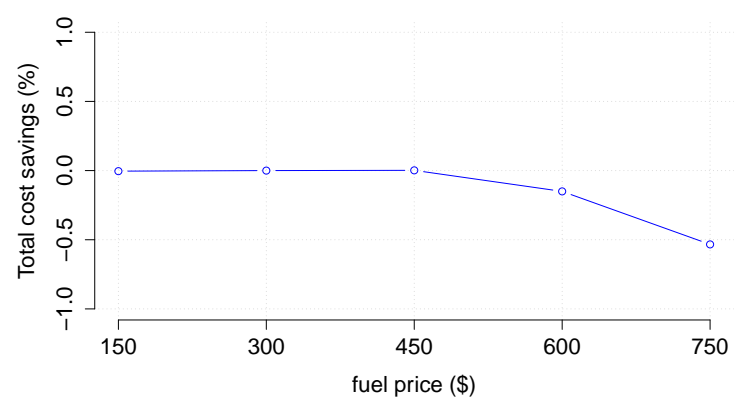

(c) Scenario A, different fuel prices

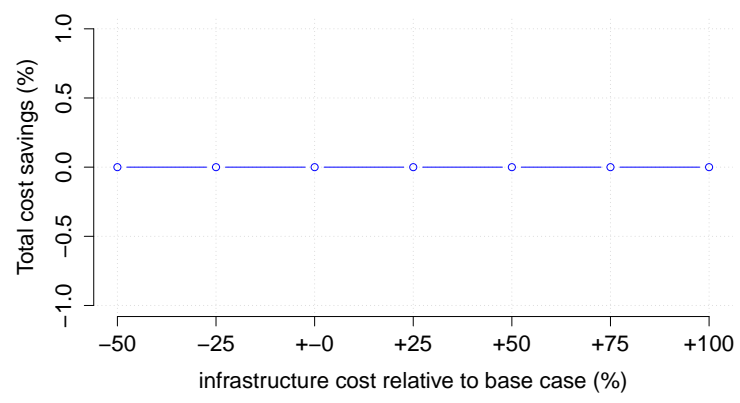

(e) Scenario A, varying infrastructure cost

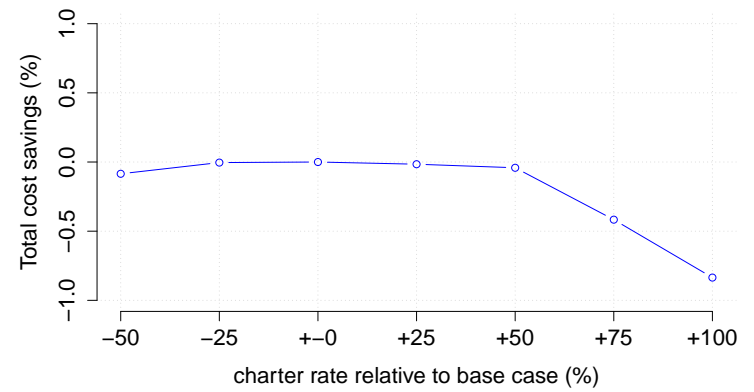

(b) Scenario B, varying charter rates

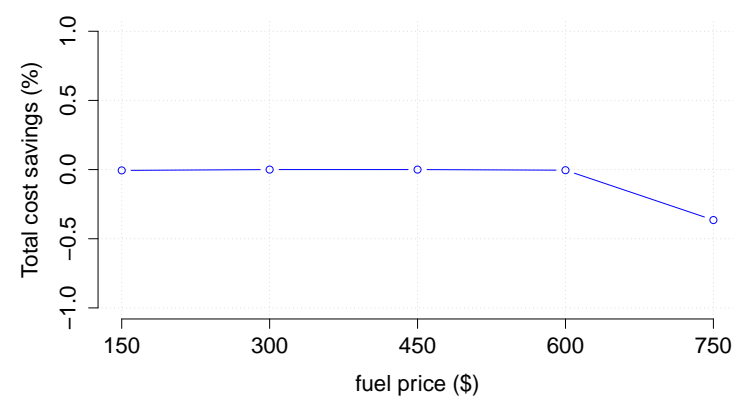

(d) Scenario B, different fuel prices

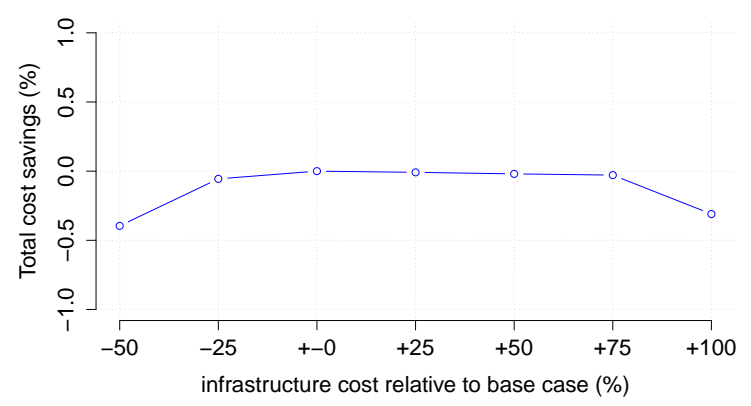

(f) Scenario B, varying infrastructure cost

Figure 12: Potential cost savings obtained through reoptimization after paramter changes compared to the optimal solution of the base case. 


\section{References}

Agra, A., Christiansen, M., and Delgado, A. (2013). Mixed integer formulations for a short sea fuel oil distribution problem. Transportation Science, 47(1):108-124.

Agra, A., Christiansen, M., Delgado, A., and Hvattum, L. M. (2015). A maritime inventory routing problem with stochastic sailing and port times. Computers and Operations Research, 61:18-30.

Andersen, M. L., Clausen, N. B., and Sames, P. C. (2013). Costs and benefits of LNG as ship fuel for container vessels. Key results from a GL and MAN joint study. Technical report, Germanischer Lloyd, MAN Diesel \& Turbo.

Andersson, H., Christiansen, M., and Desaulniers, G. (2016). A new decomposition algorithm for a liquefied natural gas inventory routing problem. International Journal of Production Research, 54(2):564-578.

Andersson, H., Christiansen, M., Desaulniers, G., and Rakke, J. G. (2015). Creating annual delivery programs of liquefied natural gas. Optimization and Engineering, pages 1-18.

Andersson, H., Christiansen, M., and Fagerholt, K. (2010). Transportation planning and inventory management in the LNG supply chain. Energy, Natural Resources and Environmental Economics, pages 427-439.

Boleda Molas, A. (2015). Maritime infrastructure optimization. Master's thesis, Technical University of Denmark, Lyngby, Denmark.

Christiansen, M. (1999). Decomposition of a combined inventory and time constrained ship routing problem. Transportation Science, 33(1):3-16.

Christiansen, M. and Fagerholt, K. (2009). Maritime inventory routing problems. In Floudas, C. A. and Pardalos, P. M., editors, Encyclopedia of Optimization, pages 1947-1955. Springer US.

Christiansen, M. and Nygreen, B. (1998). A method for solving ship routing problems with inventory constraints. Annals of Operations Research, 81(0):357-378.

Chryssakis, C., Balland, O., Tvete, H. A., and Brandsæter, A. (2014). Alternative fuels for shipping. DNV-GL strategic research \& innovation position paper. Technical report, DNVGL.

Delorme, L., Iglesias, A. S., and Perez, S. A. (2005). Sloshing loads simulation in LNG tankers with SPH. In International conference on computational methods in marine engineering.

DNV-GL (2014). Liquefied Natural Gas (LNG) Bunkering Study. Technical report, DNV-GL.

Drewry Maritime Research (2012). LNG Shipping Market. Review and Forecast. Annual Report 2012. Technical report, Drewry Maritime Research.

Furman, K. C., Song, J.-H., Kocis, G. R., McDonald, M. K., and Warrick, P. H. (2011). Feedstock routing in the exxonmobil downstream sector. Interfaces, 41(2):149-163.

Gate terminal (2014). LNG break bulk project. http://gate.nl/en/break-bulk/ lng-break-bulk-project.html. [Online; accessed 20 August 2015].

Gavory, T. and De Seze, P. E. (2009). Sloshing in membrane LNG carriers and its consequences from a designer's perspective. Proceedings of the International Offshore and Polar Engineering Conference, Proc Int Offshore Polar Eng Conf, pages 13-20. 
Goel, V., Furman, K. C., Song, J.-H., and El-Bakry, A. S. (2012). Large neighborhood search for LNG inventory routing. Journal of Heuristics, 18(6):821-848.

Grønhaug, R. and Christiansen, M. (2009). Supply chain optimization for the liquefied natural gas business. In Nunen, J. A., Speranza, M. G., and Bertazzi, L., editors, Innovations in Distribution Logistics, volume 619 of Lecture Notes in Economics and Mathematical Systems, pages 195-218. Springer Berlin Heidelberg.

Grønhaug, R., Christiansen, M., Desaulniers, G., and Desrosiers, J. (2010). A branch-andprice method for a liquefied natural gas inventory routing problem. Transportation Science, 44(3):400-415.

Halvorsen-Weare, E. E. and Fagerholt, K. (2013). Routing and scheduling in a liquefied natural gas shipping problem with inventory and berth constraints. Annals of Operations Research, 203(1):167-186.

Halvorsen-Weare, E. E., Fagerholt, K., and Rönnqvist, M. (2013). Vessel routing and scheduling under uncertainty in the liquefied natural gas business. Computers $\&$ Industrial Engineering, 64(1):290 - 301.

Hannula, S., Levander, O., and Sipilä, T. (2006). LNG cruise ferry - a truly environmentally sound ship. Technical report, Wärtsilä Corporation.

International Maritime Organization (2008). Resolution MEPC.176(58). Amendments to the annex of the protocol of 1997 to amend the international convention for the prevention of pollution from ships, 1973, as modified by the protocol of 1978 relating thereto (Revised MARPOL Annex VI).

Jokinen, R., Pettersson, F., and Saxén, H. (2015). An MILP model for optimization of a smallscale LNG supply chain along a coastline. Applied Energy, 138:423 - 431.

Kuo, J. F., Campbell, R. B., Ding, Z., Hoie, S. M., Rinehart, A. J., Sandstroem, R. E., Yung, T. W., Greer, M. N., and Danaczko, M. A. (2009). LNG tank sloshing assessment methodology - the new generation. International Journal of Offshore and Polar Engineering, 19(4):241-253.

Lloyd's Register (2012). LNG-fuelled deep sea shipping. The outlook for LNG bunker and LNG-fuelled newbuild demand up to 2025. Technical report, Lloyd's Register.

Lloyd's Register (2014). LNG Bunkering Infrastructure Survey 2014. Technical report, Lloyd's Register.

Psaraftis, H. N. and Kontovas, C. A. (2014). Ship speed optimization: Concepts, models and combined speed-routing scenarios. Transportation Research Part C: Emerging Technologies, $44: 52-69$.

Rakke, J. G., Andersson, H., Christiansen, M., and Desaulniers, G. (2015). A new formulation based on customer delivery patterns for a maritime inventory routing problem. Transportation Science, 49(2):384-401.

Rakke, J. G., Stålhane, M., Moe, C. R., Christiansen, M., Andersson, H., Fagerholt, K., and Norstad, I. (2011). A rolling horizon heuristic for creating a liquefied natural gas annual delivery program. Transportation Research Part C: Emerging Technologies, 19(5):896 - 911. Freight Transportation and Logistics (selected papers from ODYSSEUS 2009 - the 4th International Workshop on Freight Transportation and Logistics). 
Rozmarynowska, M. and Oldakowski, B. (2012). Implications of new regulation regarding sulphur content in ship's fuel on maritime transport sector within baltic sea region. Technical report, TransBaltic project, Baltic Ports Organization.

RS Platou Economic Research AS (2015). RS Platou monthly April 2015. Technical report, RS Platou Economic Research AS.

Stålhane, M., Rakke, J. G., Moe, C. R., Andersson, H., Christiansen, M., and Fagerholt, K. (2012). A construction and improvement heuristic for a liquefied natural gas inventory routing problem. Computers \& Industrial Engineering, 62(1):245 - 255.

Westney, R. (1997). The engineer's cost handbook: Tools for managing project costs. Dekker. 